\title{
What FIREs up star formation: the emergence of the Kennicutt-Schmidt law from feedback
}

\author{
Matthew E. Orr, ${ }^{1 \star}$ Christopher C. Hayward, ${ }^{2,3,1}$ Philip F. Hopkins, ${ }^{1}$ T. K. Chan, ${ }^{4}$ \\ Claude-André Faucher-Giguère, ${ }^{5}$ Robert Feldmann,,${ }^{6,7}$ Dušan Kereš, ${ }^{4}$ Norman Murray ${ }^{8}$ \\ and Eliot Quataert ${ }^{7}$ \\ ${ }^{1}$ TAPIR, California Institute of Technology, 1200 E. California Blvd., Pasadena, CA 91125, USA \\ ${ }^{2}$ Center for Computational Astrophysics, Flatiron Institute, 162 Fifth Avenue, New York, NY 10010, USA \\ ${ }^{3}$ Harvard-Smithsonian Center for Astrophysics, 60 Garden Street, Cambridge, MA 02138, USA \\ ${ }^{4}$ Department of Physics, Center for Astrophysics and Space Science, University of California at San Diego, 9500 Gilman Drive, La Jolla, CA 92093, USA \\ ${ }^{5}$ Department of Physics and Astronomy and CIERA, Northwestern University, 2145 Sheridan Road, Evanston, IL 60208, USA \\ ${ }^{6}$ Institute for Computational Science, University of Zurich, Zurich CH-8057, Switzerland \\ ${ }^{7}$ Department of Astronomy and Theoretical Astrophysics Center, University of California, Berkeley, CA 94720-3411, USA \\ ${ }^{8}$ Canadian Institute for Theoretical Astrophysics, 60 St George Street, University of Toronto, Toronto ON M5S 3H8, Canada
}

Accepted 2018 May 10. Received 2018 May 10; in original form 2017 January 6

\begin{abstract}
We present an analysis of the global and spatially resolved Kennicutt-Schmidt (KS) star formation relation in the FIRE (Feedback In Realistic Environments) suite of cosmological simulations, including haloes with $z=0$ masses ranging from $10^{10}$ to $10^{13} \mathrm{M}_{\odot}$. We show that the KS relation emerges and is robustly maintained due to the effects of feedback on local scales regulating star-forming gas, independent of the particular small-scale star formation prescriptions employed. We demonstrate that the time-averaged KS relation is relatively independent of redshift and spatial averaging scale, and that the star formation rate surface density is weakly dependent on metallicity and inversely dependent on orbital dynamical time. At constant star formation rate surface density, the 'cold and dense' gas surface density (gas with $T<300 \mathrm{~K}$ and $n>10 \mathrm{~cm}^{-3}$, used as a proxy for the molecular gas surface density) of the simulated galaxies is $\sim 0.5$ dex less than observed at $\sim$ kpc scales. This discrepancy may arise from underestimates of the local column density at the particle-scale for the purposes of shielding in the simulations. Finally, we show that on scales larger than individual giant molecular clouds, the primary condition that determines whether star formation occurs is whether a patch of the galactic disc is thermally Toomre-unstable (not whether it is self-shielding): once a patch can no longer be thermally stabilized against fragmentation, it collapses, becomes self-shielding, cools, and forms stars, regardless of epoch or environment.
\end{abstract}

Key words: instabilities - opacity - methods: numerical - galaxies: evolution-galaxies: formation-galaxies: star formation.

\section{INTRODUCTION}

Understanding star formation and its effects on galactic scales has been integral to assembling the story of the growth and subsequent evolution of the baryonic components of galaxies. Observationally, the rate at which gas is converted into stars is characterized by the Kennicutt-Schmidt (KS) relation, which is a power-law correlation between the star formation and gas surface densities in galaxies that holds over several orders of magnitude (Schmidt 1959; Kennicutt 1998; see Kennicutt \& Evans 2012, for a recent review).

Numerous studies of the KS relation have shown that star formation is inefficient on galactic scales, with only a few per cent of a galaxy's gas mass being converted to stars per galactic free-fall time (Kennicutt 1998; Kennicutt et al. 2007; Daddi et al. 2010; Genzel et al. 2010). Understanding what regulates the efficiency of star formation and results in the observed KS relation is therefore key to understanding the formation and dynamics of galaxies. Some authors (e.g. Thompson, Quataert \& Murray 2005; Murray, Quataert \& Thompson 2010; Murray 2011; Ostriker \& Shetty 2011; Faucher-Giguère, Quataert \& Hopkins 2013; Hayward \& Hopkins 
2015; Grudić et al. 2016; Semenov, Kravtsov \& Gnedin 2016) argue that star formation is locally efficient, in the sense that tens of per cent of the mass of a gravitationally bound gas clump within a giant molecular cloud (GMC) can be converted into stars on the local free-fall time, and that local stellar feedback processes - including supernovae ( $\mathrm{SNe})$, radiation pressure, photoheating, and stellar winds - must stabilize gas discs against catastrophic gravitational collapse, thereby resulting in the low global star formation efficiencies that are observed. However, others claim on both theoretical and observational grounds that star formation is locally inefficient, with only of the order of a few per cent of the mass of clumps being converted into stars on a free-fall time independent of their density (Padoan 1995; Krumholz \& Tan 2007; Lee, Miville-Deschênes \& Murray 2016).

In either scenario, the KS law is considered to be an emergent relation that holds on galactic scales and results from a complex interplay of the physical processes that trigger star formation and those that regulate it. It has also been argued and observed that the KS relation breaks down below some length- and time-scales (Onodera et al. 2010; Schruba et al. 2010; Feldmann \& Gnedin 2011; Calzetti, Liu \& Koda 2012; Kruijssen \& Longmore 2014). Calzetti et al. (2012) found that the KS relation breaks down due to incomplete sampling of star-forming molecular clouds' mass function on length-scales of less than $\sim 1 \mathrm{kpc}$. Feldmann, Gnedin \& Kravtsov (2012) claim that this breakdown on sub-kpc scales occurs due to the stochastic nature of star formation itself. Furthermore, Kruijssen \& Longmore (2014) argue that the various tracers of gas column density and star formation rate surface density require averaging over some spatial and temporal scales; consequently, when sufficiently small length-scales are probed, a tight correlation between the star formation rate surface density and the gas column density should not be observed. Understanding the scales where the KS law holds therefore informs our theories of star formation as well.

On the length-scales where the KS relation does hold, the canonical power law of the total gas relation is $\Sigma_{\mathrm{SFR}} \propto \Sigma_{\text {gas }}^{1.4}$ with $\Sigma_{\mathrm{SFR}}$ being the star formation rate surface density and $\Sigma_{\text {gas }}$ being the total gas surface density (Kennicutt 1998). However, there has been much debate regarding the power-law index of the relation and its physical origin; the previous literature has found $\mathrm{KS}$ relations ranging from highly sublinear to quadratic (Bigiel et al. 2008; Daddi et al. 2010; Genzel et al. 2010; Feldmann, Gnedin \& Kravtsov 2011, 2012; Narayanan et al. 2012; Shetty, Kelly \& Bigiel 2013; Becerra \& Escala 2014; Shetty et al. 2014a; Shetty, Clark \& Klessen 2014b). Some of the disagreement owes to the particular formulation of the KS relation considered, such as whether $\Sigma_{\mathrm{HI}+\mathrm{H}_{2}}$ (total atomic + molecular hydrogen column) or $\Sigma_{\mathrm{H}_{2}}$ (molecular column alone) is employed (e.g. Rownd \& Young 1999; Wong \& Blitz 2002; Krumholz \& Thompson 2007), with the $\Sigma_{\mathrm{H}_{2}}$ relation typically having a slope of $\sim 1$. The relation may, in principle, also depend on the star formation tracer used (e.g. H $\alpha$, far-infrared, or ultraviolet). Furthermore, there are questions as to whether the index depends on spatial resolution - even on scales larger than the length-scale below which the relation fails altogether - or if there are multiple tracks to the KS relation, each with different slopes across several decades in gas surface density (Ostriker, McKee \& Leroy 2010; Feldmann, Gnedin \& Kravtsov 2011, 2012; Liu et al. 2011; Ostriker \& Shetty 2011; Faucher-Giguère, Quataert \& Hopkins 2013).

It has also been suggested that the KS relation may evolve with redshift or have a metallicity dependence (Schaye 2004; Bouché et al. 2007; Papadopoulos \& Pelupessy 2010; Dib 2011; Gnedin \& Kravtsov 2011; Scoville et al. 2016). These are not entirely independent quantities, as metallicity generally increases as galaxies process their gas through generations of stars over cosmic timescales. Because the presence of metals results in more efficient gas cooling, and can thus aid in the transition from diffuse ionized and atomic species to dense molecular gas (Hollenbach \& Tielens 1999), Schaye (2004) and Krumholz, McKee \& Tumlinson (2009b) have argued that there is a metallicity-dependent gas surface density cut-off below which the KS relation becomes steeper. Krumholz et al. (2009b) attribute the dependence to the gas column needed to self-shield molecular gas for a given metallicity. As well, gas metallicity has been argued to weakly modulate the specific strength of stellar feedback, as SNe couple slightly less momentum into their immediate stellar surroundings since more of their energy is able to radiate away quickly (Cioffi, McKee \& Bertschinger 1988; Martizzi, Faucher-Giguère \& Quataert 2015; Richings \& Schaye 2016). Scoville et al. (2016) found evidence of shorter depletion timescales for molecular gas at higher redshifts for galaxies both on and above the 'star formation main sequence', perhaps due to the rapid accretion required to replenish the gas reservoirs.

Large-volume cosmological simulations often use the KS law as a sub-grid prescription for star formation, both because of the prohibitive computational complexity of including all of the physics relevant on the scales of star-forming regions, and their inability to resolve even the most massive giant molecular clouds $\sim 10^{6} \mathrm{M}_{\odot}$ (e.g. Mihos \& Hernquist 1994; Springel \& Hernquist 2003). Even idealized disc simulations that have resolution of the order of $100 \mathrm{pc}$, but are unable to resolve a multiphase ISM, employ star formation prescriptions that assume low star formation efficiencies a priori or implement KS laws indirectly (Li, Mac Low \& Klessen 2006; Wada \& Norman 2007; Schaye \& Dalla Vecchia 2008; Richings \& Schaye 2016). It has been shown that assuming a power-law star formation relation on the resolution scale can imprint a power-law relation of an identical slope on the galactic scale (Gnedin, Tasker \& Fujimoto 2014), demonstrating the importance of employing physically motivated sub-grid star formation prescriptions that produce kpc-scale relations with the 'correct' slope if the relevant physical processes cannot be treated directly. With advances in computing power, and the ability to execute increasingly complex simulations with more physics at higher mass resolution, cosmological simulations have only recently been able to predict the KS relation generically as a result of the physics incorporated in the simulations at the scales of molecular clouds (e.g. Hopkins, Quataert \& Murray 2011; Hopkins et al. 2013a, 2014; Agertz \& Kravtsov 2015).

Including realistic feedback physics in simulations that resolve GMC scales is critical to understanding the KS relation due to the multitude of competing physical effects spanning a wide range of scales. While some simulations have argued that the KS relation can be obtained without explicit feedback (e.g. Li, Mac Low \& Klessen 2005, 2006; Wada \& Norman 2007), these generally depend on either (a) transient and short-lived initial conditions (e.g. simulations starting from strong initial turbulence or a smooth disc, where once turbulence decays and fragmentation runs away, some additional source of 'driving' or 'GMC disruption' must be invoked), or (b) suppressing runaway fragmentation numerically (e.g. 'by hand' setting very slow star formation efficiencies at the grid scale, or inserting explicit sub-grid models for star formation calibrated to the KS relation on GMC or galaxy scales, or adopting artificial/numerical pressure or temperature floors or fixed gravitational softening in the gas that prevents densities from increasing arbitrarily). Many of these authors do acknowledge that 'feedback' is likely necessary to provide either the initial conditions or grid-scale terms in their simulations, even if not explicitly included (similarly, see e.g. Robertson \& Kravtsov 2008; Colín et al. 2010; Kuhlen et al. 2012; 
Kraljic et al. 2014). Indeed, a large number of subsequent, higher resolution numerical experiments (on scales ranging from kpc-scale 'boxes' to cosmological simulations), which run for multiple dynamical times and allow fragmentation to proceed without limit, have consistently shown that, absent feedback, the galaxy-scale KS law has a factor of $\sim 100$ higher normalization than observed (see e.g. Hopkins, Quataert \& Murray 2011; Kim, Kim \& Ostriker 2011; Ostriker \& Shetty 2011; Shetty \& Ostriker 2012; Kim, Ostriker \& Kim 2013; Dobbs 2015; Kim \& Ostriker 2015; Benincasa et al. 2016; Forbes et al. 2016; Hu et al. 2017; Iffrig \& Hennebelle 2017).

In this paper, we explore the properties and emergence of the KS relation in the FIRE $^{1}$ (Feedback In Realistic Environments) simulations (Hopkins et al. 2014). Specifically, by producing mock observational maps of the spatially resolved KS law, we investigate the form of the relation when considering several different tracers of the star formation rate and gas surface densities, and we characterize its dependence on redshift, metallicity, and pixel size. The FIRE simulations are well suited for understanding the physical drivers of the KS relation as they sample a variety of galactic environments and a large dynamic range in physical quantities (chiefly, gas and star formation rate surface densities), and they directly (albeit approximately) incorporate stellar feedback processes that may be crucial for the emergence, and maintenance of the KS relation over cosmological time-scales. In the past, they have been used to investigate the effects of various microphysics prescriptions on galaxy evolution, the formation of giant gas clumps at high redshift, and the formation of galactic discs, among other topics (Su et al. 2016; Ma et al. 2017; Oklopčić et al. 2017).

\section{SIMULATIONS AND ANALYSIS METHODS}

In the present analysis, we investigate the star formation properties of the FIRE galaxy simulations originally presented in Hopkins et al. (2014), Chan et al. (2015), and Feldmann et al. (2016), which used the Lagrangian gravity + hydrodynamics solver GIZMO (Hopkins 2013) in its pressure-energy smoothed particle hydrodynamics (PSPH) mode (Hopkins 2013). All of the simulations employ a standard flat $\Lambda$ CDM cosmology with $h \approx 0.7, \Omega_{\mathrm{M}}=1-\Omega_{\Lambda} \approx 0.27$, and $\Omega_{\mathrm{b}} \approx 0.046$. The galaxies in the simulations analyzed in this paper range in $z \approx 0$ halo masses from $9.5 \times 10^{9}$ to $1.4 \times 10^{13} \mathrm{M}_{\odot}$, and minimum baryonic particles masses $m_{\mathrm{b}}$ of $2.6 \times 10^{2}-3.7 \times 10^{5}$ $\mathrm{M}_{\odot}$. For all of the simulations, the mass resolution is scaled with the total mass such that the characteristic turbulent Jeans mass is resolved. As well, the force softening is fully adaptive, scaling with the particle density and mass as

$\delta h \approx 1.6 \mathrm{pc}\left(\frac{n}{\mathrm{~cm}^{-3}}\right)^{-1 / 3}\left(\frac{m}{10^{3} \mathrm{M}_{\odot}}\right)^{1 / 3}$,

where $n$ is the number density of the particles and $m$ is the particle mass. Consequently, the simulations are able to resolve a multiphase ISM, allowing for meaningful ISM feedback physics. This is crucial because the vast majority of star formation occurs in the most massive GMCs due to the shape of the GMC mass function (Williams \& McKee 1997).

The stellar feedback physics implemented in these simulations include approximate treatments of multiple channels of stellar feedback: radiation pressure on dust grains, supernovae (SNe), stellar winds, and photoheating; a detailed description of the stellar feedback model can be found in Hopkins et al. (2014). Star particles in

\footnotetext{
${ }^{1} \mathrm{http}: / /$ fire.northwestern.edu
}

the simulations each represent individual stellar populations, with known ages, metallicities, and masses. Their spectral energy distributions, supernovae rates, stellar wind mechanical luminosities, metal yields, etc. are calculated directly as a function of time using the STARBURST99 (Leitherer et al. 1999) stellar population synthesis models, assuming a Kroupa (2002) IMF.

In these simulations, the galaxy- and kpc-scale star formation efficiencies are not set 'by hand'. Star formation is restricted to dense, molecular, self-gravitating regions according to several criteria:

(i) The gas density must be above a critical threshold, $n_{\text {crit }} \sim 50 \mathrm{~cm}^{-3}$, in most runs (and $5 \mathrm{~cm}^{-3}$ in those from Feldmann et al. 2016).

(ii) The molecular fraction $f_{\mathrm{H}_{2}}$ is calculated as a function of the local column density and metallicity using the prescription of Krumholz \& Gnedin (2011), and the molecular gas density is used to calculate the instantaneous SFR (see below).

(iii) Finally, we identify self-gravitating regions using a sink particle criterion at the resolution scale, specifically requiring $\alpha \equiv$ $\delta v^{2} \delta h / G m_{\text {gas }}(<\delta r)<1$ on the smallest resolved scale around each gas particle ( $\delta h$ being the force softening or smoothing length).

Regions that satisfy all of the above criteria are assumed to have an instantaneous star formation rate of

$\dot{\rho}_{*}=\rho_{\mathrm{mol}} / t_{\mathrm{ff}}$,

i.e., 100 per cent efficiency per free-fall time. As a large fraction of the dense $\left(n>n_{\text {crit }}\right)$, molecular $\left(f_{\mathrm{H}_{2}} \sim 1\right)$ gas is not gravitationally bound $(\alpha>1)$ at any given time, the global star formation efficiency $\epsilon$ is less than 100 per cent $(\epsilon<1)$ despite the assumed local, instantaneous star formation efficiency per free-fall time being 100 per cent. We will show below that the KS relation, with its much lower global, time-averaged star formation efficiency $(\epsilon \lesssim 0.1)$, emerges as a result of stellar feedback preventing dense gas from quickly becoming self-bound and forming stars and disrupting gravitationally bound star-forming clumps on a time-scale less than the local free-fall time. We stress here that the emergent $\mathrm{KS}$ relation is not a consequence of the star formation prescription employed in the simulations.

In Appendix A we demonstrate this explicitly. We ran several tests restarting one of the standard FIRE simulations with varying physics and star formation prescriptions. For any reasonable set of physics, only variation in the strength of the feedback affected the galactic star formation rates, because the simulated galaxies self-regulate their star formation rates via feedback. A number of independent studies have also shown that once feedback is treated explicitly, the predicted KS law becomes independent of the resolution-scale star formation criterion (Saitoh et al. 2008; Federrath \& Klessen 2012; Hopkins, Quataert \& Murray 2012b; Agertz et al. 2013; Hopkins et al. 2013c, 2013a; Hopkins, Narayanan \& Murray 2013b; Hopkins et al. 2016).

To quantify the spatially resolved KS relation in the simulations, we analyze data from snapshots spanning redshifts $z=0-6$. The standard FIRE snapshots from Hopkins et al. (2014) and the dwarf runs in Chan et al. (2015) are roughly equipartitioned amongst redshift bins $z \sim 3-6,2.5-1.5,1.5-0.5$, and $<0.5$, whereas the snapshots of haloes from Feldmann et al. (2016) have redshifts evenly spaced between $2<z<6$ (these were run to only $z \sim 2$ ). To compare the snapshots with observational constraints of the KS relation, we made star formation rate and gas surface density maps of each snapshot's central galaxy. We summed the angular momentum vectors of the star particles in the main halo of each snapshot to determine the galaxy's rotation axis and projected along this axis to 


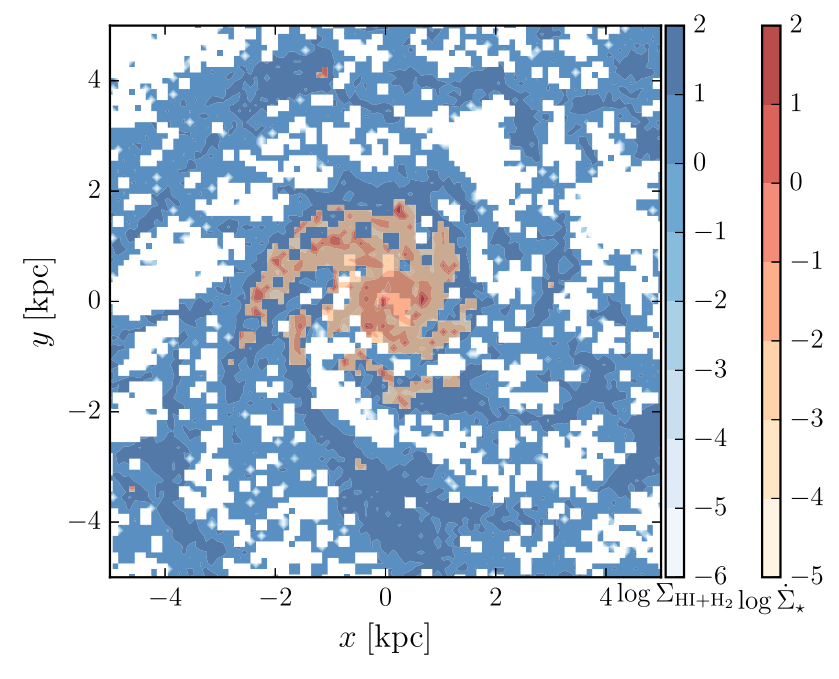

Figure 1. Example of one of our maps, made from a Milky Way-mass simulated galaxy at $z \approx 0$ (galaxy m12i from Hopkins et al. 2014), with $100 \mathrm{pc}$ pixels. Neutral hydrogen surface density, $\Sigma_{\mathrm{HI}+\mathrm{H}_{2}}\left(\mathrm{M}_{\odot} \mathrm{pc}^{-2}\right)$ and instantaneous gas star formation rate $\dot{\Sigma}_{\star}\left(\mathrm{M}_{\odot} \mathrm{yr}^{-1} \mathrm{kpc}^{-2}\right)$ are coloured in blue and red, respectively. Spiral arms and increasing density towards the galactic core are clearly visible, and the instantaneous star formation rate is seen to closely trace the densest gas structures.

generate face-on galaxy maps. The projected maps were then binned into square pixels of varying size, ranging from $100 \mathrm{pc}$ to $5 \mathrm{kpc}$ on a side. Only particles within $20 \mathrm{kpc}$ above or below the galaxy along the line of sight were included in the maps (this captures all of the star-forming gas, but excludes distant galaxies projected by chance along the same line of sight in the cosmological box). An example of the resulting maps can be found in Fig. 1, which shows maps of the neutral gas surface density and the instantaneous star formation rate surface density in the $\mathbf{m 1 2} \mathbf{i}$ simulation from Hopkins et al. (2014), at redshift $z \approx 0$ with 100 pc pixels.

Using the star particle ages, we calculated star formation rates averaged over the previous 10 and $100 \mathrm{Myr}$, correcting for massloss from stellar winds and other evolutionary effects as predicted by STARBURST99 (Leitherer et al. 1999). We also considered the instantaneous star formation rate of the gas particles (defined above). A time-averaging interval of $10 \mathrm{Myr}$ was chosen because this approximately corresponds to the time-scale traced by recombination lines such as $\mathrm{H} \alpha$, whereas UV and FIR emission traces star formation over roughly the past $100 \mathrm{Myr}$ (e.g. Kennicutt \& Evans 2012). ${ }^{2}$ The instantaneous star formation rate of the gas particles covers a larger range of star formation rates because it is not constrained at the low end directly by the mass resolution of our simulations; it is a continuous quantity intrinsic to the gas particles themselves, which is sampled at each time-step to determine if the gas particles form stars. This quantity best demonstrates the direct consequences of feedback on the gas in situ by locally tracing the star formation rate, whereas the other two SFR tracers are more analogous to observables.

The gas surface density tracers were also chosen on the basis of observable analogues, including all gas, neutral hydrogen gas (total

\footnotetext{
${ }^{2}$ Directly computing SFR indicators from the simulations (e.g. Hayward et al. 2014; Sparre et al. 2015) rather than computing the SFR averaged over the past 10 or $100 \mathrm{Myr}$ would provide a more accurate comparison with the observed KS relation, but doing so would considerably expand the scope of this work, so we leave it to a future study.
}

$\mathrm{H} \mathrm{I}+\mathrm{H}_{2}$ column, accounting for metallicity and He corrected), and 'cold and dense' gas which we specifically define here and throughout this paper as gas with $T<300 \mathrm{~K}$ and $n_{\mathrm{H}}>10 \mathrm{~cm}^{-3}$. These roughly correspond to the total gas (including the ionized component), atomic + molecular gas $\left(\mathrm{HI}+\mathrm{H}_{2}\right)$, and cold molecular gas reservoirs observed in galaxies. We have opted to use the aforementioned approximation for the molecular gas component rather than reconstruct the $f_{\mathrm{H}_{2}}$ predicted by the Krumholz \& Gnedin (2011) model (which is not output in the snapshots) as the $f_{\mathrm{H}_{2}}$ model assumes a simplified geometry at the resolution scale, which can get the local optical depth quite wrong. ${ }^{3}$ We explore the differences between the cold and dense gas tracer and the Krumholz et al. (2009b) $f_{\mathrm{H}_{2}}$ (which was the basis for Krumholz \& Gnedin 2011) in a small number of snapshots, as well as with other empirical estimators such as those adopted in Leroy et al. (2008), in Appendix B. A more detailed analysis of the true molecular fraction of the gas would require a careful radiative transfer post-processing, which we leave to a later work. Furthermore, as GIZMo lacks a detailed implementation of any chemical network, important to determining low temperature cooling, and instead uses approximate cooling tables, we may get the temperature wrong by a factor of a few below $\sim 1000 \mathrm{~K}$ (this error should have no dynamical effect in the simulations as this cool gas already effectively has no pressure compared to the bulk of the gas at higher, more reliable temperatures). Past work by Richings \& Schaye (2016) has indeed shown that metallicity and radiation field on large scales have far larger effects on star formation rates than including detailed low-temperature chemical networks.

We acknowledge that because of the rather strict density and temperature criteria, the lack of any additional considerations, e.g. to the local UV field or the geometry of the gas, and our 'low' star formation gas density threshold of $50 \mathrm{~cm}^{-3}$, we appear to underestimate the molecular gas column as measured by the cold and dense gas tracer (and other estimators calculated at the particle scale) by up to a factor of a dex, which is incidentally of the order of the uncertainty in the observational $\mathrm{CO}$ to $\mathrm{H}_{2}$ conversion factor $X_{\mathrm{CO}}$ (Bolatto, Wolfire \& Leroy 2013). This likely results in a corresponding underestimation of the local gas depletion time and overestimation of star formation efficiency. In Appendix B, we show explicitly that the 'molecular' fraction based on the "cold and dense' criteria is significantly less than the molecular gas fraction computed using two other relations for $f_{\mathrm{H}_{2}}$ versus total neutral gas surface density: that from Leroy et al. (2008) (which is based on Blitz \& Rosolowsky 2006) and the relation from Krumholz et al. (2009b) applied at the kpc-scale for total gas surface densities above their atomic-to-molecular transition thresholds. Notably, applying Krumholz et al. (2009b), with updates from Krumholz \& Gnedin 2011, at the particle scale produces a similar underestimation of the molecular gas column of $\sim 0.5$ dex like the cold and dense gas tracer. The difficulty of estimating local (at the particle scale) column depths for shielding likely contributes to the discrepancy for both the cold and dense gas tracer and Krumholz et al. (2009b) fit applied at the few-pc scale.

\footnotetext{
${ }^{3}$ For the purposes of our star formation criteria, however, this is not an issue for the vast majority of cases. Due to the steepness of the exponential attenuation of the local UV field, we care only whether, strictly speaking, the gas is optically thin or thick, but the exact value of $\tau$ is not especially important, as any optical depth $\tau \gg 1$ effectively yields $\exp (-\tau) \ll 1$, and $\tau \ll 1$ similarly yields $\exp (-\tau) \approx 1$.
} 
These empirical fits for $f_{\mathrm{H}_{2}}$, based in part on the stellar surface densities and scale heights and gas metallicity, suggest that the FIRE simulations are producing correct star formation rates for largescale properties of the ISM, e.g. mid-plane pressure, implying that the discrepancy in the cold and dense gas tracer lies with the dense end of the gas phase structure at the particle scale, and not with kpc-scale properties of the galaxies. However, we believe that the scaling relations based on the cold and dense tracer are robust, since this discrepancy results in a consistent bias in the normalization of 'cold' gas. Again, a more accurate calculation would involve radiative transfer post-processing including a chemical network, which would allow us to directly predict the molecular hydrogen fraction and $X_{\mathrm{CO}}$, which we intend to pursue in future work.

Other quantities are calculated as the mass-weighted average in each pixel, including the gas metallicity ${ }^{4} Z$, the Keplerian velocity $v_{\mathrm{c}}$, and the dynamical angular velocity $\Omega$, defined here as

$\Omega=\frac{v_{\mathrm{c}}}{R}=\frac{(G M(<R))^{1 / 2}}{R^{3 / 2}}$,

where $R$ is the galactocentric radius of the pixel and $M(<R)$ is the total mass enclosed within that radius (and $G$ is the gravitational constant). These quantities allow us to investigate the dependence of star formation on gas phase metallicity, approximate the optical depth of star-forming regions, and relate galactic dynamical times to star-forming regions.

In our analysis we treat pixels from all simulations and all times equally, unless otherwise stated. However, we wish to examine only ensembles of pixels with well-resolved SFR distributions. Recalling that each of our simulations has a fixed baryonic particle mass, $m_{\mathrm{b}}$, we discard pixels which contain $<3$ gas particles; for a pixel size $l$, this means only gas surface densities $\Sigma_{\text {gas }}>3 \times 10^{-3} \mathrm{M}_{\odot} \mathrm{pc}^{-2}\left(m_{\mathrm{b}} / 1000 \mathrm{M}_{\odot}\right)(l / \mathrm{kpc})^{-2}$ will be considered. However, in the example above $\left(m_{\mathrm{b}} \sim 1000 \mathrm{M}_{\odot}, l \sim \mathrm{kpc}\right)$, the observed Kennicutt (1998) relation gives a typical star formation surface density $\Sigma_{\mathrm{SFR}} \sim 10^{-7} \mathrm{M}_{\odot} \mathrm{yr}^{-1} \mathrm{kpc}^{-2}$ at this minimum $\Sigma_{\text {gas }}$, so in $\sim 10 \mathrm{Myr}$, the expected number of $m_{\mathrm{b}} \sim 1000 \mathrm{M}_{\odot}$ star particles formed will be just 0.001 . Obviously, the distribution of star formation rates will not, then, be resolved (even if the simulations capture the mean star formation rate correctly, the discrete nature of star formation means only 1 in 1000 pixels will have a star particle, while 999 have none). Thus, to ensure that the pixels we examine from each simulation at a given gas surface density have a well-resolved SFR distribution, we adopt the following criteria: (1) we first calculate the mean $\Sigma_{\text {SFR }}$ per pixel from each simulation, for all their pixels with a given number of gas particles (fixed $\Sigma_{\text {gas }}$ ); (2) we estimate the average number $\left\langle N_{\star}(\Delta t)\right\rangle$ of star particles this would produce in the time $\Delta t(10$ or $100 \mathrm{Myr}$, as appropriate); (3) if this is $<1\left(=N_{\min , \star}\right)$, we discard all pixels which contain this number or fewer gas particles. For the example above, for $\Delta t=10 \mathrm{Myr}$ $(100 \mathrm{Myr})$, this requires $>500(>50)$ gas particles per pixel for a 'resolved' star formation rate. We have repeated this exercise using instead the observed KS relation (instead of the predicted one), to estimate the resolved thresholds, and find it gives nearly identical results. We have also verified that changing the threshold $N_{\min , \star}$ by an order of magnitude in either direction does not change any of our conclusions here; we note too that the average star formation rates from low-resolution simulations continue to agree well with our higher resolution simulations down to $\left\langle N_{\star}(\Delta t)\right\rangle$ as low as $\sim 0.001$.

\footnotetext{
${ }^{4}$ In this paper, we take solar metallicity to be $Z_{\odot} \approx 0.0142$ when scaling
} metallicities (Asplund et al. 2009).
We believe it is important to reassert that pixels with no star formation contribute to all of the plotted points in our KS relation. We are discarding sets of pixels (those with and without star formation) that do not have at least one young star particle on average at a given gas surface density, to ensure that all of our plotted data points are drawn from well-resolved distributions of star formation (including zero star formation) at a given gas surface density.

We are careful that this prescription does not introduce bias into our star formation distributions at a given gas surface density. If we were to consider the distribution of depletion time $\left(\Sigma_{\text {gas }} / \dot{\Sigma}_{\star}\right)$ across all gas surface densities, this method would bias us towards shorter depletion time by discarding all the pixels below the gas surface density that definitely produces at least one new star particle in the past 10 (or 100) Myr. However, we are investigating the distribution of SFRs in bins of gas surface density for ensembles of pixels from a number of individual galaxy simulations. To do so, we examine many snapshots from each individual simulation and consider whether the SFR distribution is well sampled by the ensemble of pixels from all of those snapshots at a given gas surface density. If at that gas surface density in the whole ensemble of pixels from that single simulation, there are at least $N_{\star}$ (we have chosen one here ${ }^{5}$ ) new star particles produced on average, then we believe we are able to say something meaningful about the distribution of star formation rates in that bin of gas surface density for that ensemble of pixels. In combining only the sets of pixels from individual simulations with resolved SFR distributions at a given gas surface density, we thus avoid biasing our aggregated SFR (and by extension, depletion time) distributions in each bin of gas surface density.

\subsection{Observational data}

Comparing with observations, we compiled resolved KS observations from a large number of papers at various resolution scales commensurate with our mock observational maps. For our $1 \mathrm{kpc}$ 'fiducial' scale maps of the KS relation, we compare our neutral gas surface density results with a combination data from Kennicutt et al. (2007), Bigiel et al. (2008), and Genzel et al. (2010); we compare our $1 \mathrm{kpc}$ 'cold and dense' gas surface density results with the appropriate $\mathrm{H}_{2}$ results from these studies, as well as those from Verley et al. (2010). For exploring the effects of pixel size, we also used these molecular gas data to compare with our 500-pc maps, as these observations had varying resolution scales ranging from $500 \mathrm{pc}$ to slightly larger than $1 \mathrm{kpc}$. For our galaxy-averaged $5 \mathrm{kpc}$ maps, we used data from Kennicutt (1998), Kennicutt et al. (2007), Genzel et al. (2010), Shapiro et al. (2010), Wei et al. (2010), Freundlich et al. (2013), Tacconi et al. (2013), and Amorín et al. (2016). Finally, for our highest resolution investigations at $100 \mathrm{pc}$, we compared with high-resolution observations from Blanc et al. (2009) and Onodera et al. (2010). For exploring the star formation efficiency in this work, in the form of the Elmegreen-Silk relation, we compare our $1 \mathrm{kpc}-$ scale maps with observations from Kennicutt (1998) and Daddi et al. (2010). No distinction is made between the many estimators of SFR used in the aforementioned papers; they are simply taken at face value. However, we re-calibrate $X_{\mathrm{CO}}$ in the observationally

\footnotetext{
${ }^{5}$ We have confirmed that this approach does not bias the average SFR surface density values by repeating the analysis requiring only an average of $N_{\star}=0.001$ star particles per pixel. In this case, however, the distribution of SFR surface density at a given gas surface density is poorly sampled because of Poisson noise.
} 
inferred $\Sigma_{\mathrm{H}_{2}}$ data points across all the aforementioned resolved KS studies with an interpolation function taken from Narayanan et al. (2012), of the form $X_{\mathrm{CO}}=\min \left[4,6.75 \times W_{\mathrm{CO}}^{-0.32}\right] \times 10^{20} \mathrm{~cm}^{-2} /(\mathrm{K}$ $\mathrm{km} \mathrm{s}^{-1}$ ), independent of metallicity. ${ }^{6}$ To correct the quoted $\Sigma_{\mathrm{HI}+\mathrm{H}_{2}}$ measurements, we decomposed the total column into atomic and molecular components (the latter then being corrected in the manner of the $\Sigma_{\mathrm{H}_{2}}$ 's) using data in the references themselves, where available, and assuming a molecular fraction fit from Leroy et al. (2008) where necessary. We explore the effects of variations of the assumed $X_{\mathrm{CO}}$ on the (dis)agreement with our simulations in Appendix $\mathrm{C}$, finding an $\sim 0.5$ dex uncertainty due to the uncertainty in $X_{\mathrm{CO}}$. In the case of the Elmegreen-Silk relation observations from Kennicutt (1998) and Daddi et al. (2010), being unable to separate out the dynamical times, we recalibrate Kennicutt (1998) only to a constant $X_{\mathrm{CO}}=2 \times 10^{20} \mathrm{~cm}^{-2} /\left(\mathrm{K} \mathrm{km} \mathrm{s}^{-1}\right)$, consistent with Bigiel et al. (2008). Data from Daddi et al. (2010) have not been altered due to the extensive efforts made therein to calibrate $X_{\mathrm{CO}}$ across their data set.

\section{KS RELATION IN THE SIMULATIONS}

\subsection{Dependence of the KS relation on star formation and gas tracers}

Fig. 2 demonstrates how a KS-like power-law relation selfconsistently emerges (recall that the assumed instantaneous star formation efficiency of dense, gravitationally bound 'molecular' gas is 100 per cent per local free-fall time) in the FIRE simulations irrespective of specific choice of star formation or gas tracer. Two of our star formation rate tracers, the $10 \mathrm{Myr}$-averaged and gas instantaneous star formation rates, yield very similar KS relations. The points denote the median value of the star formation rate distribution in that gas surface density bin. The thick (thin) error bars in Fig. 2 denote the $25-75$ per cent (5-95 per cent) inclusion interval in the distribution of the star formation rates of pixels in that bin of gas surface density, effectively the $\pm 1 \sigma( \pm 2 \sigma)$ scatter. The $1 \sigma$ scatter of our 10 Myr-averaged SFR, neutral gas, KS relation is $\sim 0.4$ dex, in line with quoted scatters from Bigiel et al. (2008) and Leroy et al. (2013).

More restrictive gas tracers (e.g. taking gas with $T<300 \mathrm{~K}$ and $n_{\mathrm{H}}>10 \mathrm{~cm}^{-3}$, instead of all atomic + molecular gas) yield shallower power-law slopes. This is intuitive because by placing more restrictions on the gas column, we are taking pixels at a given star formation rate and moving them to lower gas surface densities (to the left) by reducing what gas contributes to the overall gas column density. The restrictions are non-linear: at high surface densities, the gas is predominately molecular, and added restrictions do little to change the participating gas column, whereas at low surface densities, relatively little of the gas column may remain after making these additional cuts. Little difference is seen between the star formation distributions in $\Sigma_{\mathrm{SFR}}-\Sigma_{\text {gas }}$ space when considering the surface densities of all gas (including the ionized component) versus neutral gas (first and second columns of Fig. 2) because the contribution of ionized gas to the total gas column is small in regions where significant star formation is occurring. In contrast, there is a marked change in slope of the KS relation when moving from the surface density of neutral hydrogen gas to that of cold and dense gas $\left(T<300 \mathrm{~K}, n_{\mathrm{H}}>10 \mathrm{~cm}^{-3}\right)$, with the slope shifting from $\sim 1.7$

\footnotetext{
${ }^{6}$ Though their full interpolation function included a metallicity dependence, we assume solar metallicity for simplicity.
}

to $\sim 1.2$ for the gas instantaneous star formation rate. This is due to the fact that significant amounts of star formation can occur in 'small' pockets of molecular gas, relative to the overall gas column, yielding a shallower slope than when considering neutral gas.

The neutral gas surface density KS relation in the FIRE simulations is consistent with the corresponding spatially resolved observational data, as represented by the shaded regions and points in the panels of Fig. 2. No observational range has been included for 'all gas' observations as this is not typically observed; nevertheless, our data suggest that little change would be evident, as again, ionized gas does not usually contribute significantly to the column of starforming gas. There is significant, though consistent, disagreement between the simulations and observations for our cold and dense gas surface density because our 'cold and dense' appears to underestimate the expected molecular gas surface density by $\sim 0.5-1$ dex. In Appendix B, we explore the uncertainty in the molecular gas mass estimate by comparing the cold and dense gas tracer with other empirical estimators for the molecular fraction of our pixels, such as the dependence on the mid-plane gas pressure used in Leroy et al. (2008), adapted from earlier work (Blitz \& Rosolowsky 2006), and the self-shielding-based method from Krumholz et al. (2009b). There, we see that the cold and dense gas tracer (which is calculated on a per-particle basis) appears to underpredict molecular gas fractions by $\sim 0.5-0.7$ dex across surface densities of $1-100 \mathrm{M}_{\odot}$ $\mathrm{pc}^{-2}$ compared to the kpc-averaged empirical estimators. This suggests that although our star formation rates are appropriate given the large-scale properties of the ISM (e.g. mid-plane pressure and dust opacity), we are underpredicting the mass of gas at the highest densities, either by converting it into stars too quickly as it crosses our star formation density threshold or by incorrectly approximating the cooling and shielding properties of the densest gas. However, as this underprediction appears to be consistent across the range of gas surface densities explored, we believe the form of the KS relation to be robust and have added arrows indicating this $\sim 0.5$ dex underestimate whenever results based on the cold and dense gas tracer are presented. Exploring this further is beyond the scope of this work and is the subject of a forthcoming study forward modelling dense gas tracers in FIRE-2.

Interestingly, the distribution of star formation rates in the FIRE simulations overlaps with that of damped Ly $\alpha$ systems (DLAs) observed at high redshift by Rafelski et al. (2016). Though we appear to see analogues to these systems at $1 \mathrm{kpc}^{2}$ pixel sizes, we leave it to a future work to investigate the detailed physical properties of these systems.

\subsubsection{Elmegreen-Silk relation (alternative KS law)}

Alternatively, in Fig. 3, we probe the global efficiency of gas turning into stars in a dynamical time according to

$\dot{\Sigma}_{\star}=\epsilon \Sigma_{\text {gas }} \Omega$,

where $\epsilon$ represents the 'star formation efficiency' on kpc scales; this relation is known as the Elmegreen-Silk relation (Elmegreen 1997; Silk 1997). We see systematic agreement with the neutral hydrogen gas surface density Elmegreen-Silk relation in the FIRE simulations compared to observations where $\epsilon$ ranges between $10^{-3}$ and 1 . We find kpc-averaged star formation efficiencies of $\epsilon \sim 0.01-0.1$ consistently for our entire range of star formation rate surface density. Dashed black lines indicate constant efficiencies between 0.01 and 1. Without feedback, one would expect to see $\epsilon \sim 1$.

Our efficiencies for the molecular gas formulation of the Elmegreen-Silk relation are likely overestimated by as much as 


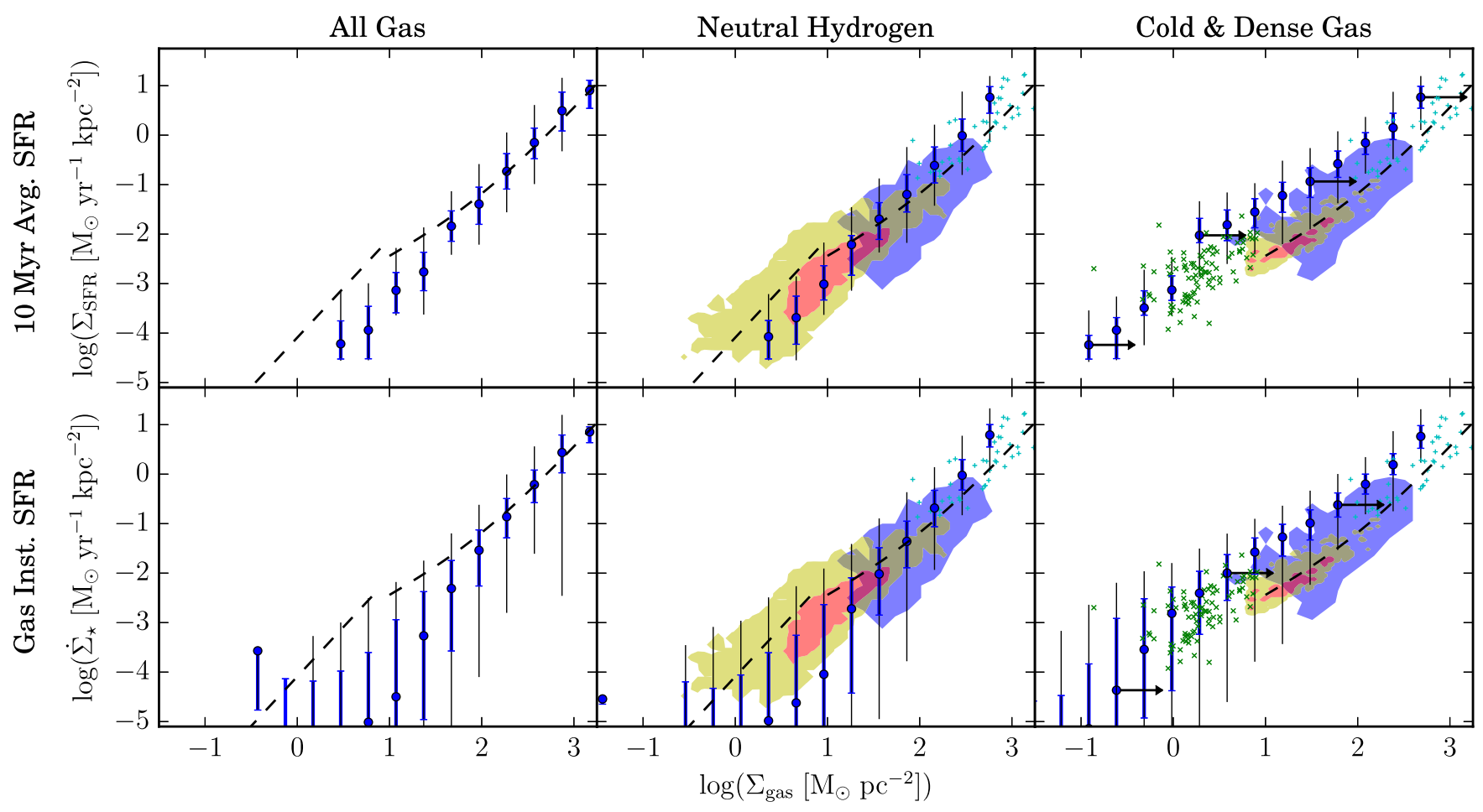

Figure 2. KS relation in the FIRE runs in $1 \mathrm{kpc}^{2}$ pixels, binned by $\Sigma_{\text {gas }}$, for several gas and star formation 'tracers'. Neutral hydrogen is $\sim \Sigma_{\mathrm{HI}+\mathrm{H}_{2}}$, and cold and dense gas includes particles with $T<300 \mathrm{~K}$ and $n_{\mathrm{H}}>10 \mathrm{~cm}^{-3}\left(\sim \Sigma_{\mathrm{H}_{2}}\right)$. The gas instantaneous star formation rate is calculated directly from the gas particles in each pixel, whereas the $10 \mathrm{Myr}$ average star formation rate is calculated from the young star particles in each pixel. Median values of the pixel distribution are plotted in bins of gas surface density, with thick (thin) error bars denoting the 25-75 per cent (5-95 per cent) range of resolved star formation in the bins. In both the middle and right columns, the yellow and red shaded regions denote observational data from Bigiel et al. (2008), and the blue shaded region illustrates the region spanned by the resolved observations from Kennicutt et al. (2007). Observations of high-redshift dusty star-forming galaxies from Genzel et al. (2010) (cyan +'s) and molecular gas data from Verley et al. (2010) (green x's, right column only) are also included. All observations have been re-calibrated with the Narayanan et al. (2012) variable $X_{\mathrm{CO}}$ interpolation function, as described in Section 2.1. The star formation relations derived in Sections 4.1 and 4.2 are plotted with dashed black lines, using the fiducial values assumed there and $\Sigma_{\star}=10^{2} \mathrm{M}_{\odot} \mathrm{pc}^{-2}$. The simulations' kpc-scale total gas and neutral hydrogen KS relations are consistent with observational constraints within the uncertainties. The simulation relation computed using the cold and dense tracer is systematically offset from the observed molecular gas relation; this may be due to the cold and dense tracer underestimating the molecular fraction (see the text and Appendix B). The lower limit error bars in the cold and dense gas panels indicate the 0.5 dex uncertainty in our conservative estimator of molecular gas.

1 dex, at efficiencies between a few and a few tens of percent, because of our systematic underestimation of the mass of 'cold and dense' gas (see also the discussion at the end of Section 3.1 and in Appendix B). However, since this is likely consistent across gas surface densities, we believe that the relative constancy of global star formation efficiency $\epsilon$ across $\Sigma_{\text {gas }}$ is robust. Error bars of 0.5 dex (which are likely conservative) indicate this underestimation in the cold and dense gas panels. Even so, we find consistency at the high end of the observed efficiencies using the molecular gas formation of the Elmegreen-Silk relation, where our molecular fraction finally converges to near unity.

\subsubsection{Myr-averaged star formation rate}

In Fig. 4, we see a clear flattening of the 100 Myr-averaged star formation rate surface density relative to the $10 \mathrm{Myr}$ average, for neutral hydrogen columns at low gas surface densities, $\Sigma_{\text {gas }} \lesssim$ $1 \mathrm{M}_{\odot} \mathrm{pc}^{-2}$. This is ascribable to effects discussed in Sections 3.2 and 3.4, where individual or small numbers of young star particles are scattered into regions of very low gas surface density that are not actually forming stars. Moreover, dynamical changes in starforming regions over the averaging period (100 Myr) cause gas complexes to dissipate and produce small numbers of star particles left in now-diffuse galactic environments. At high gas surface densities, $\Sigma_{\text {gas }}>10 \mathrm{M}_{\odot} \mathrm{pc}^{-2}$, the $100 \mathrm{Myr}$ average star formation rate surface densities agree well with the shorter time-scale estimators.

\subsection{Pixel size dependence}

The KS relation that we find in the FIRE simulations does not appear to have a significant dependence on pixel size (i.e. map resolution) for pixels with sufficiently resolved gas and star formation rate tracers ( $\gtrsim$ few gas/star particles per pixel), as shown in Fig. 5. Over the range of pixel sizes we investigate, $100 \mathrm{pc}$ to $5 \mathrm{kpc}(0.01-25$ $\mathrm{kpc}^{2}$ ), the slope of the power law varies only weakly between $\sim 1$ and $\sim 4 / 3$. At the low end of the relation in $\Sigma_{\text {gas }}$, we expect the scatter to grow as Poisson statistics become important when only a few star particles are present in the pixels on average. However, because we exclude poorly sampled pixels, this simply manifests as a lower limit to the plotted $\Sigma_{\text {gas }}$ for smaller pixels sizes (see Section 2).

In terms of slope, our simulated relations agree with the observed relations for the various pixel sizes considered, but again, the simulated and observed relations are systematically offset, likely because the 'cold and dense' gas tracer systematically underestimates the 


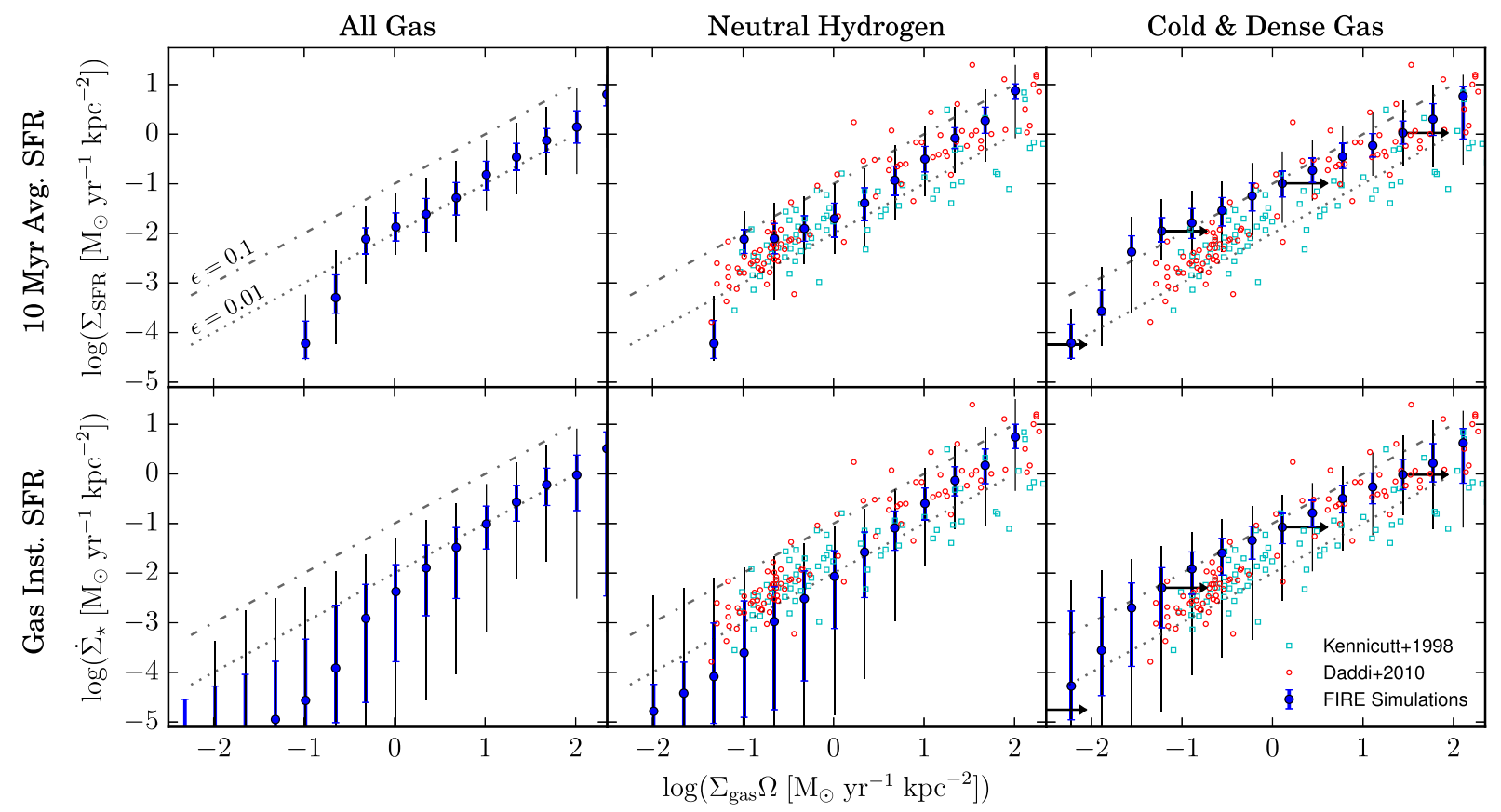

Figure 3. Elmegreen-Silk relation in the FIRE runs in $1 \mathrm{kpc}^{2}$ pixels, in the style of Fig. 2. Lines of constant star-forming efficiency are plotted, with $\epsilon=(0.01$, 0.1). Unfilled cyan squares and red circles are observational data from Kennicutt (1998) and Daddi et al. (2010), respectively. The data from Kennicutt (1998) have been recalibrated to an $X_{\mathrm{CO}}$ value consistent with Bigiel et al. (2008), but those of Daddi et al. (2010) are unaltered. The simulated galaxies have kpc-scale star formation efficiencies increasing from $\sim 1$ per cent (all gas) to $\sim 10$ per cent (cold and dense gas) as denser gas tracers are selected.

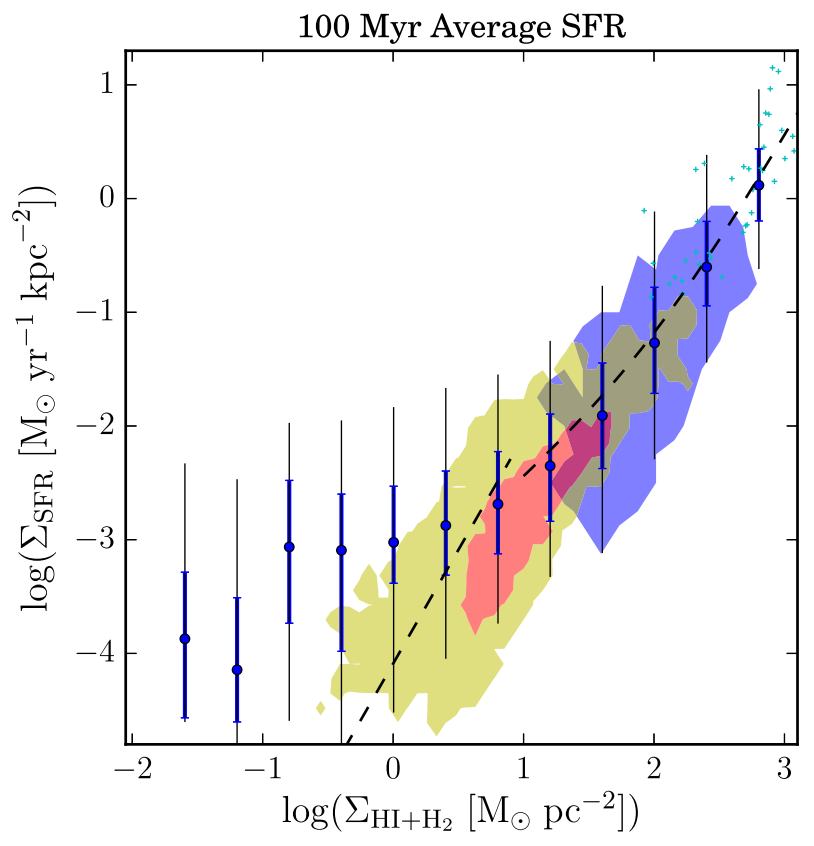

Figure 4. KS relation in the FIRE simulations for the $100 \mathrm{Myr}$-averaged star formation rate in $1 \mathrm{kpc}^{2}$ pixels, as Fig. 2. The observational data shaded regions and points for the neutral (atomic + molecular) gas are those from Kennicutt et al. (2007), Bigiel et al. (2008), and Genzel et al. (2010) as described in Fig. 2, measured with $\sim 10$ Myr tracers. At high $\Sigma_{\text {gas }}$, the $\sim 100 \mathrm{Myr}$ average SFRs agree well with the $\sim 10 \mathrm{Myr}$ observations (and by extension our $\sim 10 \mathrm{Myr}$-averaged SFRs). At low $\Sigma_{\text {gas }}$, the $\Sigma_{\mathrm{SFR}}$ from the $\sim 100 \mathrm{Myr}$ tracer flattens. This appears to stem from a breakdown in the correlation between 100 Myr-old stars and the observed gas tracers, either from migration or other dynamical effects (e.g. mergers or strong outflow events). column density of molecular gas (by $\sim 0.5-1$ dex) relative to that computed using the fits of Leroy et al. (2008) and Krumholz et al. (2009b), as already discussed above. In addition to the shaded regions shown in previous plots, we also compare directly with the results of Schruba et al. (2010) and Bolatto et al. (2011). Schruba et al. (2010) compare the KS relation found for varying aperture scales in M33, centred either on $\mathrm{H} \alpha$ or $\mathrm{CO}$ peaks. ${ }^{7}$ Their results vary weakly with pixel size, except at their smallest aperture scale $\sim 75$ pc. Similarly, Bolatto et al. (2011) observed the KS relation in the Small Magellanic Cloud (SMC), and averaged their results with $200 \mathrm{pc}$ and $1 \mathrm{kpc}$ apertures to investigate its dependence on averaging scale. Their results are also consistent with our simulations, considering that the 'cold and dense' tracer underestimates the molecular fraction by as much as a dex for surface densities above $10 \mathrm{M}_{\odot} \mathrm{pc}^{-2}$. We see, however, a slightly steeper KS relation at pixel sizes of 100 and $500 \mathrm{pc}$ (their data at $200 \mathrm{pc}$ lie between these scales) and a slightly shallower relation at kpc scales than Bolatto et al. (2011).

To compare with the global KS relation observed by a number of studies (see Section 2.1 for references), we summed the total $10 \mathrm{Myr}$ star formation rate and cold and dense gas mass in each map and then divided these sums by the area circumscribed by the stellar half-mass radius calculated for each snapshot in order to produce analogous global KS results. Our global molecular KS relation is nearly identical in form to that observed, but like other results involving our cold and dense gas tracer, our gas surface densities appear to be underestimated by $\sim 0.5-0.7$ dex for $\Sigma_{\text {mol }} \gtrsim$ $1 \mathrm{M}_{\odot} \mathrm{pc}^{2}$.

At our smallest pixel size (100 pc), however, none of our simulations are able to adequately sample star formation at gas surface

\footnotetext{
${ }^{7}$ Schruba et al. (2010) do not tile M33 with their apertures, but this does not appear to matter except at their smallest aperture scales.
} 


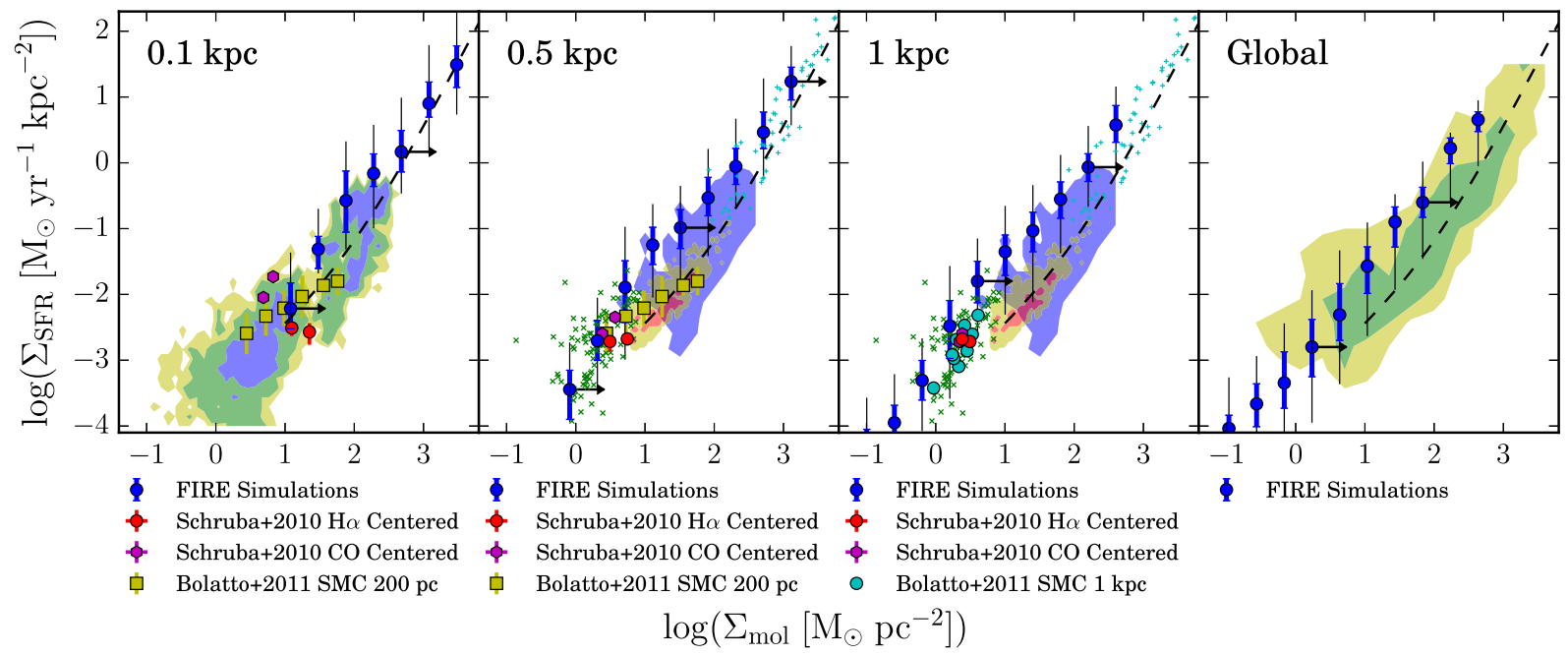

Figure 5. Pixel size dependence of the molecular KS relation in the FIRE simulations, compared with selected observations. $\Sigma_{\text {mol }}$ is the surface density of cold and dense gas $\left(T<300 \mathrm{~K}, n_{\mathrm{H}}>10 \mathrm{~cm}^{-3}\right) ; \Sigma_{\mathrm{SFR}}$ is the 10 Myr-averaged star formation rate surface density. Plotted points and error bars are as in Fig. 2. Shaded regions and points (small green x's and cyan +'s) for both $500 \mathrm{pc}$ and $1 \mathrm{kpc}$ denote $\mathrm{H}_{2}$ observations, as described in Fig. 2, because a number of the source observations lie between those two resolutions. In the $0.1 \mathrm{kpc}$ panel, shaded regions (yellow, green, blue) denote the individual inclusion contours $(100,90,50$ per cent) from Blanc et al. (2009) and Onodera et al. (2010). For the global KS relation panel, the yellow (green) shaded areas are the 70 per cent (50 per cent) inclusion regions for global molecular-KS observations compiled from the references listed in Section 2.1. To calculate our global KS relation, we sum the SFR and cold and dense gas mass values in the map and divide the sums by $\pi R_{1 / 2 \star}^{2}$, where $R_{1 / 2 \star}$ is the stellar half-mass radius. All observations have been re-calibrated with the Narayanan et al. (2012) variable $X_{\mathrm{CO}}$ interpolation function, as described in Section 2.1. Lower limit error bars indicate our $\sim 0.5$ dex uncertainty in molecular gas mass using the conservative cold and dense gas estimator. Various points explicitly enumerated below the panels correspond to observations of M33 from Schruba et al. (2010) and the SMC from Bolatto et al. (2011), testing the scale dependence of the KS relation in those environments. The star formation relations derived in Section 4.1 are plotted with dashed black lines as in Fig. 2. For each pixel size, there is a threshold in $\Sigma_{\text {mol }}$ below which star formation is poorly resolved given even our highest mass resolution, as described in Section 2. Above this threshold, the KS relation in the simulated galaxies exhibits no systematic trend with pixel size despite dynamical processes that might be expected to break down the correlations between young stars and gas on small scales (prominent at small pixels sizes).

densities $\lesssim 10 \mathrm{M}_{\odot} \mathrm{pc}^{-2}$, given our mass resolution; this regime is where observations exhibit the largest scatter. At least three processes cause the correlation of the star-forming gas and young star particles to break down on scales less than $l \sim 500$ pc. (1) The relative velocities between star-forming gas and the young stars they produce cause them to wander into different pixels, thus they become uncorrelated on a pixel-by-pixel basis, when $v_{\mathrm{p}} \sim l / \Delta t$. For $100 \mathrm{pc}$ pixels and $10 \mathrm{Myr}$ time bins, this is a relative velocity of only $\sim 10 \mathrm{~km} \mathrm{~s}^{-1}\left(1 \mathrm{~km} \mathrm{~s}^{-1}\right.$ for $\Delta t \sim 100 \mathrm{Myr}$ ), so we would expect significant scatter to arise from this effect at the smallest pixel sizes. (2) Dynamical processes affecting gas and star particles, like dispersion of GMCs, major mergers, or $\mathrm{SNe}$, over the time bin (i.e. $10-100 \mathrm{Myr}$ ) cause greater fluctuations from the power-law average as pixel size decreases. (3) When considering small $(<1 \mathrm{kpc})$ pixels, scatter is caused by the stochastic nature of the star formation in the simulations. Above $\sim 10 \mathrm{M}_{\odot} \mathrm{pc}^{-2}$, the simulated and observed relations again agree in terms of slope, but the normalization is offset by $\sim 1$ dex due to the cold and dense gas tracer underestimating the molecular gas fraction.

\subsection{Redshift independence}

We find no significant redshift dependence of either the KS or Elmegreen-Silk relations in the FIRE galaxies. The insensitivity to redshift in the simulations can be seen in Fig. 6 and the top panel of Fig. 7, where the snapshots are coloured by redshift bin $(z$ $<0.5,0.5-1.5,1.5-2.5,3-6)$ and the 10 Myr-averaged $\Sigma_{\mathrm{SFR}}$ and neutral gas surface density are considered. Similarly, no redshift dependence was seen for the cold and dense gas version of either relation; consequently, and due to the extensively discussed issues with the cold and dense tracer, these results are not shown. Some scatter is seen in the average values between redshift bins, but any dependence on redshift is much smaller than the range of the data itself. The absence of any redshift dependence persists for all measures of star formation rate. Though the absolute amount of star formation varies with redshift, the correlation between gas column and star formation rate surface density, and star formation efficiency, remains consistent.

\subsection{Metallicity dependence}

We see in the bottom panel of Fig. 7 evidence of a weak dependence on metallicity for the KS relation in the FIRE runs. For all neutral gas surface densities, more metal-rich gas exhibits elevated star formation rates, with an admittedly large scatter (there is significant overlap in $\Sigma_{\mathrm{SFR}}$ range for various $Z$ bins). At low gas surface densities $\left(\sim 1-10 \mathrm{M}_{\odot} \mathrm{pc}^{-2}\right)$, the strength of the metallicity dependence appears to be consistent with the predictions of Krumholz et al. (2009b) and Dib (2011). Interestingly, none of our forms of the KS law exhibit a notable metallicity-dependent cut-off in star formation, as some models predict (Krumholz et al. 2009b). For our 10 and 100 Myr-averaged star formation rates, this may be an issue of adequately sampling star formation rates in the "cutoff' regime of $\dot{\Sigma}_{\star} \sim 10^{-(3-4)} \mathrm{M}_{\odot} \mathrm{yr}^{-1} \mathrm{kpc}^{-2}$. However, for the well-resolved instantaneous star formation rate, the form of the star formation relation does not change at all for any of the metallicity bins in the 'cut-off' regime that Krumholz et al. (2009b) find. The instantaneous star formation rate tracer, as with the averaged 


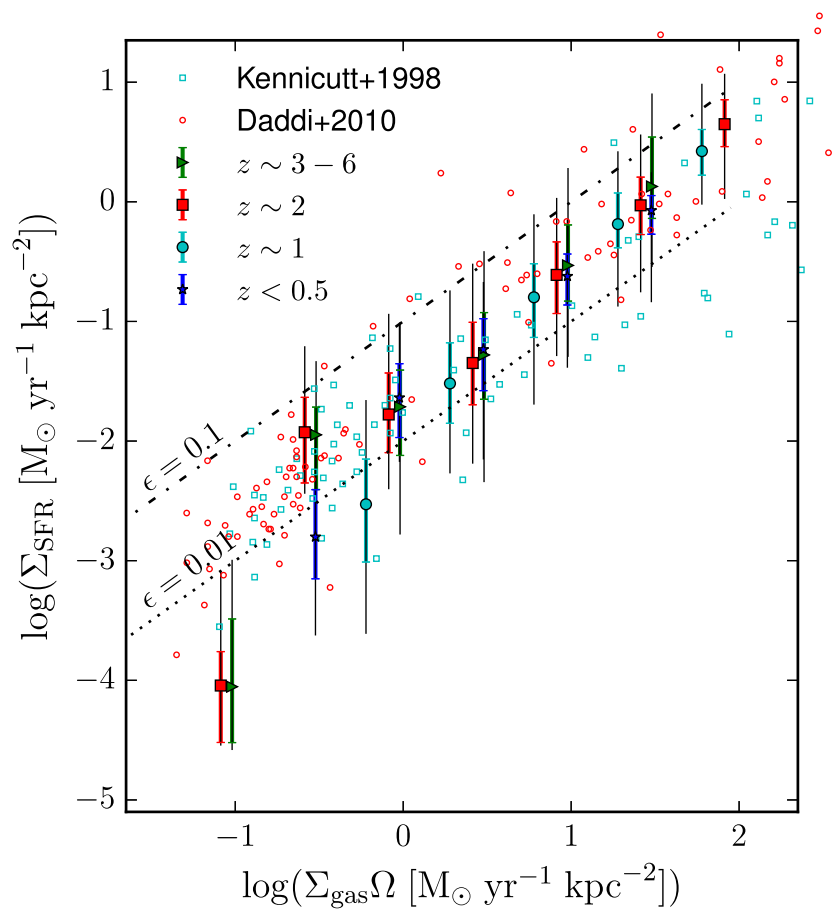

Figure 6. Elmegreen-Silk relation binned in redshift at $1 \mathrm{kpc}^{2}$ pixel size. Median values of the $10 \mathrm{Myr}$ average $\Sigma_{\mathrm{SFR}}$ are plotted in bins of $\Sigma_{\mathrm{HI}+\mathrm{H}_{2}} \Omega$, in the style of Fig. 2. Observations from Kennicutt (1998) (unfilled cyan squares) and Daddi et al. (2010) (unfilled red circles), in addition to dotted lines representing constant star formation efficiencies $\epsilon$, are included. No significant dependence on redshift is seen: the range of data in each bin is greater than any systematic difference between bins.

star formation rate tracers, presents higher star formation rates for metal-enriched gas even at these low gas surface densities, but the change is smooth, rather than a 'threshold' effect. The metallicity dependence does not appear to be strongly dependent on gas surface density, and star formation rates remain consistently positively correlated with metallicity above $10 \mathrm{M}_{\odot} \mathrm{pc}^{-2}$, differing from the model of Dib (2011), which argues for a negative correlation owing to the metallicity dependence of pre-supernova feedback (e.g. momentum coupling in winds).

Fig. 8 illustrates the strength of the metallicity dependence. Binning pixels by gas surface density $\Sigma_{\text {gas }}$ and normalizing by the average star formation rate in each $\Sigma_{\text {gas }}$ bin, we find that star formation rate surface density increases weakly with metallicity below approximately solar metallicity and considerably stronger above solar metallicity across all $\Sigma_{\text {gas }}$ bins. This presentation of the data normalizes out the $\Sigma_{\text {gas }}$ dependence to highlight the much weaker $Z$ dependence. A by-eye fit of a power law with $\dot{\Sigma}_{\star} \propto Z^{\prime 3 / 14}$ for sub-solar metallicities and $\propto Z$ above solar metallicity is plotted as a dashed black line. In the sub-solar regime, this slope is much shallower than the slope derived later in Section 4.2 but of the order of the predicted metallicity dependence of SNe feedback's momentum injection (ranging from $\sim 1 / 10$ to $3 / 14$; Cioffi et al. 1988; Martizzi et al. 2015). ${ }^{8}$ A lack of a strong dependence on

\footnotetext{
${ }^{8}$ As described in Hopkins et al. (2014), when SNe explode in regions such that their cooling radii will be unresolved (common in some of the lower resolution simulations here with particle masses $\gtrsim 10^{4} \mathrm{M}_{\odot}$ ), the ejecta are assigned a terminal momentum based on the detailed individual explosion
}

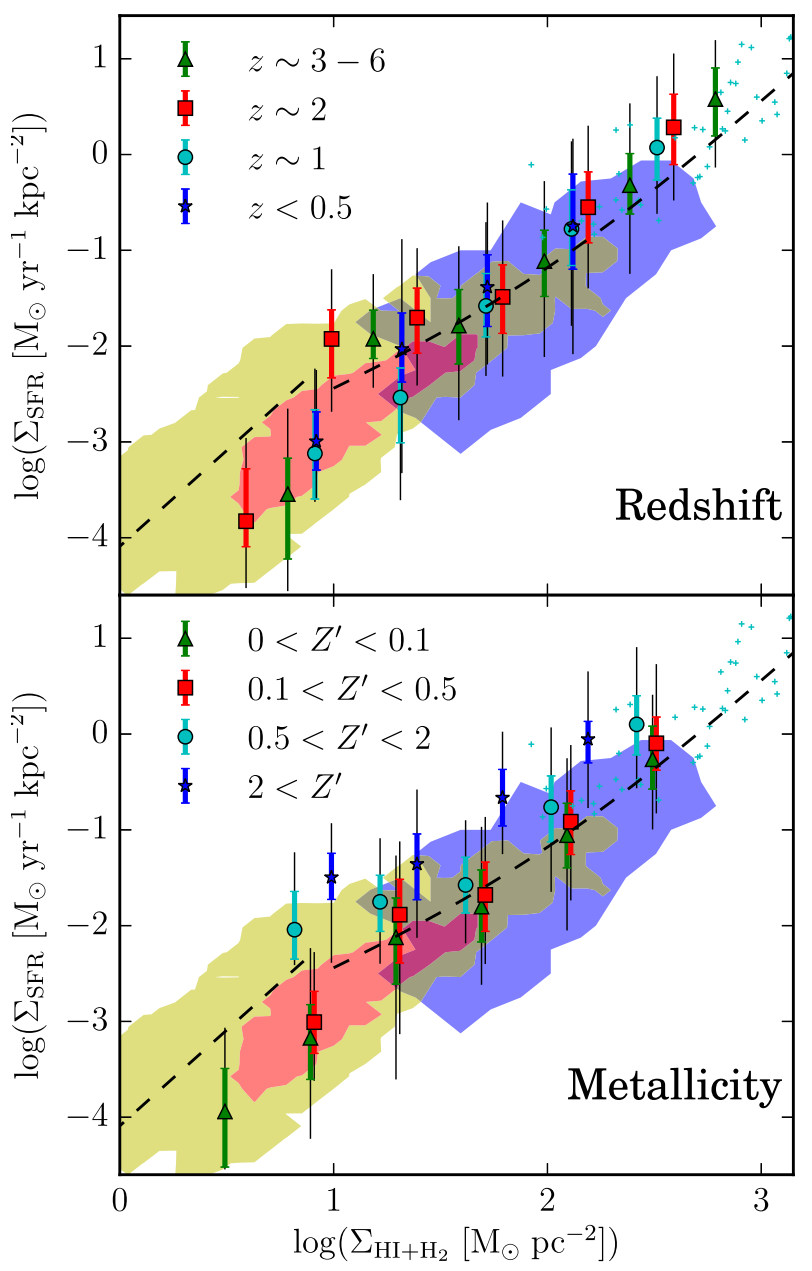

Figure 7. KS relation binned in redshift and metallicity at $1 \mathrm{kpc}^{2}$ pixel size. In both panels, within their respective redshift and metallicity bins, median values of the $10 \mathrm{Myr}$ average $\Sigma_{\mathrm{SFR}}$ are plotted in bins of $\Sigma_{\mathrm{HI}+\mathrm{H}_{2}}$, in the style of Fig. 2. Shaded regions and small cyan triangles denote representative observations from Kennicutt et al. (2007), Bigiel et al. (2008), and Genzel et al. (2010), and dotted lines represent the derived star formation relations, all as described in Fig. 2. Top panel: Simulation snapshots binned by redshift, with markers denoting different epochs. No significant dependence on redshift is seen - the range of data in each bin is greater than any systematic difference between them. Bottom panel: Pixels from snapshots binned by gas metallicity, with markers indicating intervals in $Z^{\prime}=Z_{\text {gas }} / Z_{\odot}$. A weak positive correlation between $\Sigma_{\mathrm{SFR}}$ and metallicity is seen at all $\Sigma_{\mathrm{HI}+\mathrm{H}_{2}}$, though the dependence is weak compared to the scatter in each gas bin. No metallicity-dependent cut-off is evident.

gas surface density appears to indicate that the metallicity dependences of star formation due to pre-supernova feedback effects are subdominant compared to that of supernova feedback in the FIRE simulations (Dib, Hony \& Blanc 2017). Above approximately solar metallicity, a stronger, nearly linear dependence appears. This dependence is more consistent with that derived in Section 4.2, but the reasons for its appearance are unclear and warrant future investigation. Though the scatter within bins is quite large, the weak (and stronger) dependences are rather robust across all gas surface densi-

models from Cioffi et al. (1988), which scale as $p_{\mathrm{t}} \propto Z^{3 / 14}$. However, the metallicity dependence in Fig. 8 persists if we restrict only to our highest resolution simulations. 


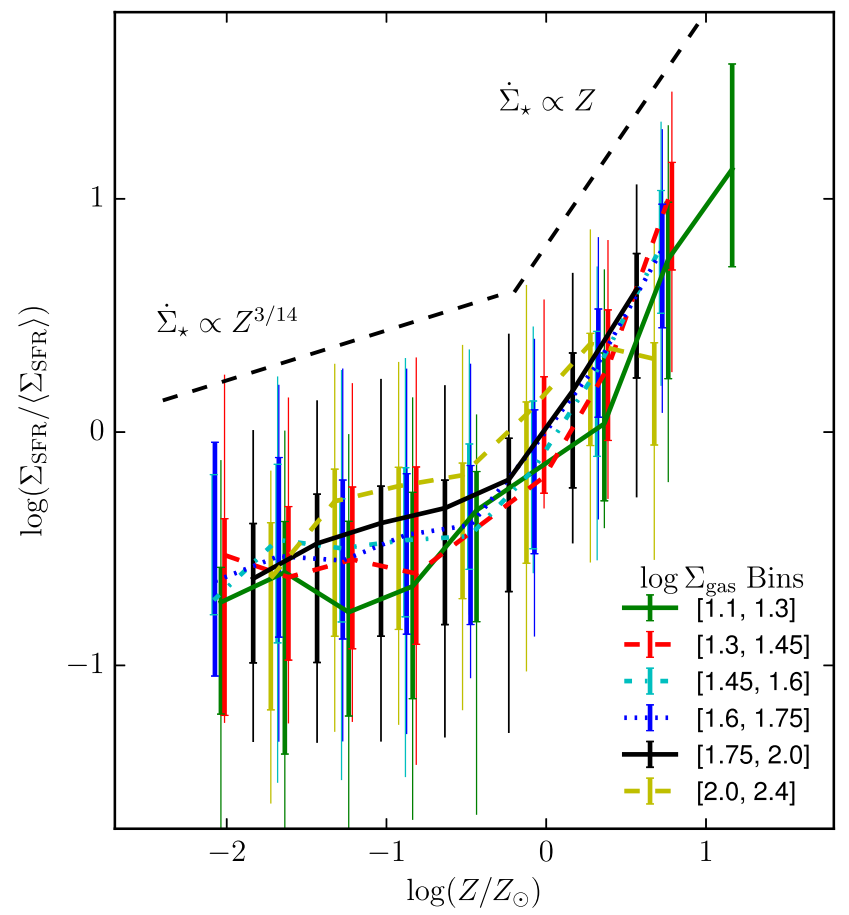

Figure 8. Star formation rate dependence on metallicity, binned by $\Sigma_{\mathrm{HI}+\mathrm{H}_{2}}$ at $1 \mathrm{kpc}^{2}$ pixel size. Points are median values of the $10 \mathrm{Myr}$ average $\Sigma_{\mathrm{SFR}}$ normalized by the average $\Sigma_{\mathrm{SFR}}$ for a given bin in $\mathrm{Z} / \mathrm{Z}_{\odot}$ in each $\Sigma_{\mathrm{HI}+\mathrm{H}_{2}}$ bin. Thick (thin) error bars denote the 25-75 per cent ( 5-95 per cent) range for resolved star formation in each bin. A weak dependence on metallicity is seen for all gas surface densities for sub-solar metallicities, as demonstrated by the dashed black line of slope $\Sigma_{\mathrm{SFR}} \propto Z^{3 / 14}$. But a stronger, nearly linear, dependence is seen for all gas surface densities above solar metallicity values, evidenced by the dashed line with slope $\Sigma_{\mathrm{SFR}} \propto Z$.

ties. A similar dependence on metallicity was found in the cold and dense gas version of Figs 7 and 8, and for brevity, we do not include them.

\subsection{Dependence on dynamical time}

In a similar manner to Fig. 8, we investigate the dependence of SFR surface density on $\Omega\left(=1 / t_{\text {dyn }}\right)$ in Fig. 9. Again normalizing the 10 Myr SFR surface density to the average SFR surface density within bins of $\Sigma_{\text {gas }}$, we see a strong nearly linear dependence of SFR surface density on $\Omega$, as expected both for a turbulently supported ISM, as discussed in Section 4.1 (see equation 6), and the thermally supported regime discussed in Section 4.2 (see equation 13). Interestingly, this persists for all gas surface densities, connecting the low- and high-gas-surface-density regimes. The dependence on $\Omega$ appears to be weaker at higher gas surface densities, which may point to an increasing prevalence of 'turbulent' Toomre stability (see equation 9 , with no explicit $\Omega$ dependence).

\section{PHYSICAL INTERPRETATION}

On the scales of tens or hundreds of millions of years, it is possible to understand star formation as an equilibrium process (on galactic scales) in which the inputs of either momentum injection from stellar feedback (at high gas surface density) or energy from photoheating (at low gas surface density) balance gravitational collapse.

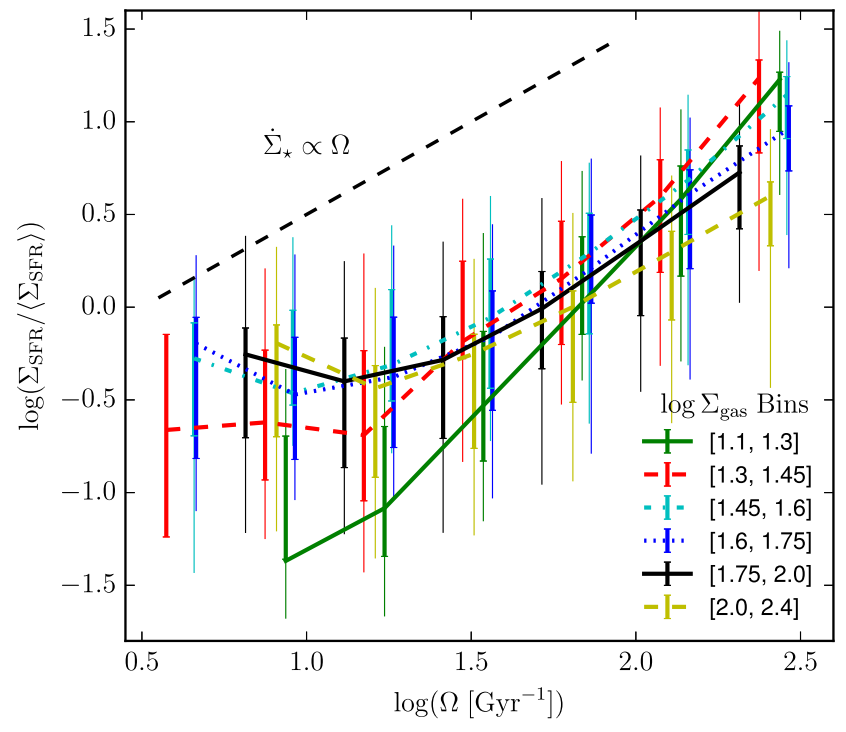

Figure 9. SFR surface density versus $\Omega$ (1/dynamical time), binned by $\Sigma_{\mathrm{HI}+\mathrm{H}_{2}}$ at $1 \mathrm{kpc}^{2}$ pixel size. Points are median values of the $10 \mathrm{Myr}$ average $\Sigma_{\mathrm{SFR}}$ normalized by the average $\Sigma_{\mathrm{SFR}}$ for a given bin in $\Omega$ in each $\Sigma_{\mathrm{HI}+\mathrm{H}_{2}}$ bin. Thick (thin) error bars denote the 25-75 per cent ( $5-95$ per cent) range for resolved star formation in each bin. A strong inverse dependence on dynamical time is seen for all gas surface densities despite considerable scatter, as demonstrated by the dashed black line with slope $\Sigma_{\mathrm{SFR}} \propto \Omega=1 / t_{\mathrm{dyn}}$.

\subsection{High-gas-surface-density regime}

In our analysis, there is a marked transition in the star formation rate distribution at gas column densities above $\Sigma_{\text {gas }} \sim 100 \mathrm{M}_{\odot}$ $\mathrm{pc}^{-2}$. Above this threshold, almost all the gas forms stars on or very near the KS power law. Here, supernova feedback becomes an increasingly important mechanism for injecting momentum into the ISM, as the massive young stars produced are embedded in dense molecular environments to which they can effectively couple.

A star formation relation can be derived in the limit in which the ISM is supported against gravitational collapse by turbulent pressure (Ostriker \& Shetty 2011; Faucher-Giguère et al. 2013; Hayward \& Hopkins 2015; Torrey et al. 2016; Dib et al. 2017). Here, stellar feedback injects momentum into the ISM at a rate per area proportional to $\dot{\Sigma}_{\star}\left(P_{\star} / m_{\star}\right)$, where $\left(P_{\star} / m_{\star}\right)$ is the characteristic momentum injected per mass of young stars formed, and is dissipated in the mass of nearby gas per area $\Sigma_{\text {gas }}$ on some characteristic time-scale related to the coherence time of the turbulent eddies $t_{\text {eddy }}$, where $t_{\text {eddy }} \sim l_{\text {eddy }} / \sigma_{\text {eddy }}, l_{\text {eddy }}$ being the spatial scale of the eddy and $\sigma_{\text {eddy }}$ the turbulent velocity $\sigma_{\mathrm{T}}$. As we are considering an approximately disc-like environment for star formation in the high-gas-surface-density regime, the largest eddies will likely have length-scales of the order of the disc scale height $\mathrm{H}$ (Martizzi et al. 2016), so $l_{\text {eddy }} \sim \mathrm{H} \sim \sigma_{\mathrm{T}} / \Omega$, with $\sigma_{\mathrm{T}}$ being the turbulent velocity and $\Omega$ being the local orbital dynamical frequency. We are concerned with the largest eddies, which contain most of the turbulent energy. Hence, the time-scales of turbulent energy dissipation scale as $t_{\text {eddy }} \approx t_{\text {diss }} \sim \mathrm{H} / \sigma_{\mathrm{T}} \sim \Omega^{-1}$. Equating these rates of turbulent momentum injection and momentum dissipation in gas ${ }^{9}$, we

${ }^{9}$ Here lies a direct connection to the no-feedback isolated disc simulations. If star formation is equated to the mass flux of gas into a 'dense' regime times a fixed efficiency, and that dense gas is then prevented from further 
find

$\dot{\Sigma}_{\star}\left(P_{\star} / m_{\star}\right) \approx \sigma_{\mathrm{T}} \Sigma_{\text {gas }} / t_{\text {diss }}$,

substituting in our relations, this yields a star formation rate of

$\dot{\Sigma}_{\star} \approx \sigma_{\mathrm{T}} \Omega \Sigma_{\text {gas }}\left(\frac{P_{\star}}{m_{\star}}\right)^{-1}$.

Relating $\sigma_{\mathrm{T}} \Omega$ back to the disc surface density with a modified Toomre- $Q$ parameter (Toomre 1964),

$Q=\frac{\kappa \sqrt{c_{\mathrm{s}}^{2}+\sigma_{\mathrm{T}}^{2}}}{\pi G \Sigma_{\mathrm{disc}}}$,

where $\kappa$ is the epicyclic frequency $\sim \sqrt{2} \Omega$ for galactic potentials with flat rotation curves, $c_{\mathrm{s}}$ is the sound speed, and $\Sigma_{\mathrm{disc}} \approx \Sigma_{\mathrm{gas}}+\Sigma_{\star}$ is the disc surface density. Here we include the self-gravity contribution from the collisionless stellar component of the disc, which is correct up to some order unity pre-factor. Assuming that we are turbulently rather than thermally supported, $\sqrt{c_{\mathrm{s}}^{2}+\sigma_{\mathrm{T}}^{2}} \approx \sigma_{\mathrm{T}}$. Substituting in this Àturbulent' Toomre-Q, we find

$\dot{\Sigma}_{\star} \approx \frac{\pi}{\sqrt{2}} G Q\left(\frac{P_{\star}}{m_{\star}}\right)^{-1} \Sigma_{\text {gas }}\left(\Sigma_{\text {gas }}+\Sigma_{\star}\right)$.

Adopting a fiducial value for $\left(P_{\star} / m_{\star}\right)$ of $\sim 3000 \mathrm{~km} \mathrm{~s}^{-1}$ (e.g. Ostriker \& Shetty 2011; Faucher-Giguère et al. 2013; Kim \& Ostriker 2015; Martizzi et al. 2015), this yields

$$
\begin{array}{r}
\dot{\Sigma}_{\star} \approx 3.3 \times 10^{-2}\left(\frac{P_{\star} / m_{\star}}{3000 \mathrm{~km} / \mathrm{s}}\right)^{-1}\left(\frac{\Sigma_{\mathrm{gas}}\left(\Sigma_{\mathrm{gas}}+\Sigma_{\star}\right)}{10^{4} \mathrm{M}_{\odot}^{2} \mathrm{pc}^{-4}}\right) Q \\
\mathrm{M}_{\odot} \mathrm{yr}^{-1} \mathrm{kpc}^{-2} .
\end{array}
$$

For the gas-dominated regime, where $\Sigma_{\text {gas }} \gg \Sigma_{\star}$, we recover a quadratic relation for star formation. Similarly, should the stellar component dominate, as may be the case in stellar systems with older populations, a linear law in $\Sigma_{\text {gas }}$ is found; this appears to be in good agreement with the slope of the KS relation seen in the FIRE runs.

The observed weak metallicity dependence seen in Figs 7 and 8 , combined with the result shown in the lower left-hand panel of Fig. A1, which shows explicitly that the star formation rate varies with the strength of feedback, can be partly explained by a weak dependence of the final momentum injection from $\mathrm{SNe}$ feedback on the metallicity of the surrounding gas, e.g. $\left(P_{\star} / m_{\star}\right)^{-1} \sim Z^{0.114}$ (Martizzi et al. 2015).

The KS relation predicted by a turbulence-supported model assuming that the stellar surface density $\Sigma_{\star} \gg \Sigma_{\text {gas }}$, with its approximately linear power-law slope, agrees remarkably well with the KS relation at moderately high surface density in the FIRE simulations. Remarkably - given the simplicity of the derivation - when equation (9) is used on a pixel-by-pixel basis to predict star formation rates from $\Sigma_{\mathrm{HI}+\mathrm{H}_{2}}$ and $\Sigma_{\star}$, the predicted rates are nearly identical to the $10 \mathrm{Myr}$-averaged and instantaneous star formation rates, extending down to $\Sigma_{\mathrm{HI}+\mathrm{H}_{2}} \approx 10^{-1} \mathrm{M}_{\odot} \mathrm{pc}^{-2}$.

\subsection{Low-gas-surface-density regime}

At the other extreme of galactic environments, we consider the lowgas-surface-density regime in which gas is supported by thermal rather than turbulent - pressure. We expect this transition to occur

star formation, we see that we expect a $\mathrm{KS}$ relation to arise with the correct slope and normalization, albeit a contrived one. for $\Sigma_{\text {gas }} \lesssim 10 \mathrm{M}_{\odot} \mathrm{pc}^{-2}$ (see Schaye 2004; Ostriker et al. 2010; Hayward \& Hopkins 2015, for details). In this regime, a star formation equilibrium rate can be derived by balancing photoheating from young stars with gas cooling. At extremely low gas surface densities, where $\Sigma_{\text {gas }} \ll 1 \mathrm{M}_{\odot} \mathrm{pc}^{-2}$, the metagalactic UV background itself may become the predominant source of heating, requiring no star formation at all to maintain a thermal pressure equilibrium, providing a physical SFR floor (Schaye 2004; Ostriker et al. 2010).

As derived in Ostriker et al. (2010) as an 'outer-disc' law, we can balance photoheating with radiative gas cooling. For ionizing and photoelectric photons dominating the gas heating, the heating rate per area is

$\frac{\dot{\mathrm{E}}_{\text {heat }}}{l^{2}}=\frac{f_{\mathrm{abs}} \beta \mathrm{L}_{\star}}{l^{2}}=f_{\mathrm{abs}} \beta \epsilon c^{2} \dot{\Sigma}_{\star}$,

where $f_{\text {abs }}(\lesssim 1)$ is the fraction of the emitted photoheating photons absorbed by surrounding gas, $\beta \sim 0.1$ is the fraction of ionizing radiation emitted by young stars (Leitherer et al. 1999), and $\epsilon \sim 4 \times 10^{-4}$ is the fraction of rest-mass energy radiated by stars in their lifetimes. On the other hand, the cooling rate per area is

$\frac{\dot{\mathrm{E}}_{\mathrm{cool}}}{l^{2}}=\frac{\Lambda n_{\mathrm{e}} n_{\mathrm{i}} V}{l^{2}} \approx \frac{\Lambda Z n_{\mathrm{g}} \Sigma_{\mathrm{gas}}}{\mu}$,

with $\Lambda \sim 10^{-22} \mathrm{erg} \mathrm{s}^{-1} \mathrm{~cm}^{-3}$ being the net cooling rate (Robertson \& Kravtsov 2008); $n_{\mathrm{e}}, n_{\mathrm{i}}$, and $n_{\mathrm{g}}$ being the electron, ion, and gas number densities, respectively; and $V \sim l^{2} h$ being the volume of gas considered. Equating the heating and cooling rates, we find

$\dot{\Sigma}_{\star} \approx \frac{\Lambda Z n_{\mathrm{g}} \Sigma_{\mathrm{gas}}}{f_{\mathrm{abs}} \mu \beta \epsilon c^{2}}$.

Furthermore, we have $n_{\mathrm{g}}=\rho_{\text {gas }} / \mu \approx \Sigma_{\text {gas }} / 2 h \mu$ and $h \approx c_{\mathrm{s}} / \Omega$ in the thermally supported limit, as $\sqrt{c_{\mathrm{s}}^{2}+\sigma_{\mathrm{T}}^{2}} \approx c_{\mathrm{s}}$. Thus, we have $n_{\mathrm{g}} \approx$ $\Sigma_{\mathrm{gas}} \Omega / 2 c_{\mathrm{s}} \mu$, and $\dot{\Sigma}_{\star}$ becomes

$\dot{\Sigma}_{\star} \approx \frac{\Lambda Z \Omega \Sigma_{\mathrm{gas}}^{2}}{2 f_{\mathrm{abs}} \mu^{2} \beta \epsilon c^{2} c_{\mathrm{s}}}=\frac{\Sigma_{0} Z \Omega}{f_{\mathrm{abs}}}\left(\frac{\Sigma_{\mathrm{gas}}}{\Sigma_{0}}\right)^{2}$,

where $\Sigma_{0} \approx 2 \mu^{2} \beta \epsilon c^{2} c_{\mathrm{s}} / \Lambda \approx 4 \mathrm{M}_{\odot} \mathrm{pc}^{-2}$, assuming $T=10^{4} \mathrm{~K}$, for which $c_{\mathrm{s}} \approx 12 \mathrm{~km} \mathrm{~s}^{-1}$. Scaling this to approximately Milky Way values $\left(\Omega \approx v_{\mathrm{c}} / R \approx 220 \mathrm{~km} \mathrm{~s}^{-1} / \mathrm{R}, Z \approx \mathrm{Z}_{\odot}\right)$, we have

$$
\begin{array}{r}
\dot{\Sigma}_{\star} \approx 1.3 \times 10^{-3}\left(\frac{Z}{\mathrm{Z}_{\odot}}\right)\left(\frac{10 \mathrm{kpc}}{R}\right)\left(\frac{\Sigma_{\mathrm{gas}}}{\Sigma_{0}}\right)^{2} \frac{1}{f_{\mathrm{abs}}} \\
\mathrm{M}_{\odot} \mathrm{yr}^{-1} \mathrm{kpc}^{-2} .
\end{array}
$$

The star formation rate relation for a thermally supported ISM has the same $\dot{\Sigma}_{\star} \propto \Sigma_{\text {gas }}^{2}$ dependence as the turbulently supported highgas-surface-density regime when the gas surface density dominates, but with an added dependence on $Z$ and $\Omega$. This is similar to the relations found by Ostriker et al. (2010) and Krumholz et al. (2009b) at low surface densities, with their dependence on metallicity. The scaling here is in good agreement with the FIRE runs at low gas surface densities for the $\Sigma_{\mathrm{HI}+\mathrm{H}_{2}}$ tracer but differs from the shallower relations found by some observational studies (Bigiel et al. 2010; Roychowdhury et al. 2015). For low column densities, where $f_{\text {abs }}$ $\ll 1$ (and usually $f_{\mathrm{g}} \ll 1$ ), the fraction of absorbed photoheating photons may go as the optical depth and thus the gas surface density $f_{\text {abs }} \propto(1-\exp (-\tau)) \approx \tau \propto \Sigma_{\text {gas }} Z^{\prime}$, reducing the low-gas-surfacedensity relation to $\dot{\Sigma}_{\star} \propto \Sigma_{\text {gas }}$, degenerate in form with the relation derived for the high-gas-surface-density regime when $f_{\mathrm{g}} \ll 1$, which may explain the aforementioned shallower observations and weak $Z$ dependence seen in Fig. 8. Similarly to the turbulently supported regime derivation, comparing the predictive ability of equation (14) 


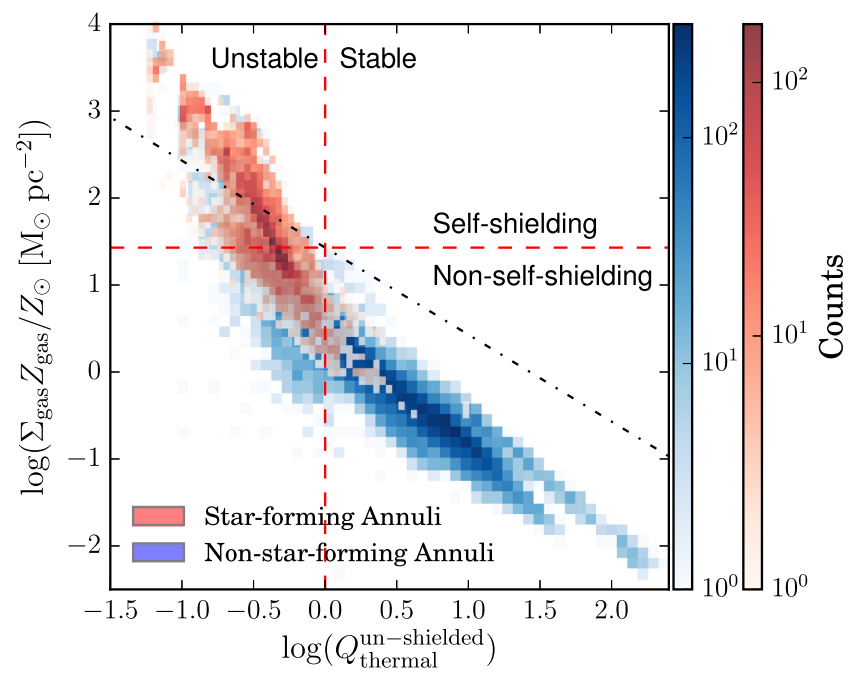

Figure 10. Comparison of whether self-shielding or gravitational instability determines the onset of efficient star formation (see Section 4.3). For each radial annulus in each galaxy (500 pc wide annuli, at each time from $z=0.5$ to 0 ), we measure $\tilde{Q}_{\text {thermal }}^{\text {un-shielded }}$ (equation 15) and $\Sigma_{\text {gas }} Z_{\text {gas }} / Z_{\odot}$. We plot a heat map of the number of pixels with each value of $\tilde{Q}_{\text {thermal }}^{\text {un }}$-shied and $\Sigma_{\text {gas }} Z_{\text {gas }} / Z_{\odot}$, colour coded so star-forming annuli are red (mean $\Sigma_{\star}>10^{-3}$ $\mathrm{M}_{\odot} \mathrm{kpc}^{-2} \mathrm{yr}^{-1}$ ) and non-star-forming annuli are blue. $\tilde{Q}_{\text {thermal }}^{\text {un-shielded }}$ is the Toomre- $Q$ parameter if the gas were purely thermally supported with $T=10^{4}$ $\mathrm{K}$; this indicates whether the gas could be thermally stabilized against gravitational instabilities if it were not self-shielding. $\Sigma_{\text {gas }} Z_{\text {gas }} / Z_{\odot}$ is a proxy for optical depth, approximating whether or not the gas is self-shielding to ionizing radiation. Vertical and horizontal dotted red lines indicate the $Q=1$ stability threshold and the self-shielding threshold derived in Krumholz et al. (2009b), respectively. Black dotted line shows the track of varying $\Sigma_{\text {gas }}$ at fixed $Z^{\prime}$ and $\Omega$. The onset of star formation clearly occurs around $\tilde{Q}_{\text {thermal }}^{\text {un-shied }} \sim 1$, even though the annuli are not self-shielding, i.e. gravitational instability initiates collapse, which then produces dense self-shielding clumps.

using pixel-by-pixel values of $Z, \Omega$, and $\Sigma_{\mathrm{HI}+\mathrm{H}_{2}}$ with the measured star formation rates, very close agreement is found for $\Sigma_{\mathrm{HI}+\mathrm{H}_{2}} \lesssim 10$ $\mathrm{M}_{\odot} \mathrm{pc}^{-2}$, the regime in which the relation is expected to apply (Hayward \& Hopkins 2015).

The transition between linear and quadratic dependences of the star formation rates on the gas surface density in the KS relation, for both the low- and high-gas-surface-density regimes, may explain some of the 'kinks' seen in the relation by various observers (e.g. Bigiel et al. 2008).

\subsection{Star formation thresholds}

There remains the question of what, physically, fires up star formation in the simulations in the first place. Figs 10 and 11 address this question.

Consider a radial annulus of a smooth gas disc at some large radius $R$. At sufficiently large $R$ and low densities, the disc is not self-shielding to UV radiation, and even the metagalactic UV background is sufficient to maintain the disc at warm temperatures $T \sim 10^{4} \mathrm{~K}$. The thermal Toomre- $Q$ parameter at this temperature:

$\tilde{Q}_{\text {thermal }}^{\text {un-shielded }} \equiv \frac{\kappa c_{\mathrm{s}}\left(10^{4} \mathrm{~K}\right)}{\pi G \Sigma_{\text {disc }}} \approx 1.2\left(\frac{\Omega}{\mathrm{Gyr}^{-1}}\right)\left(\frac{\mathrm{M}_{\odot} \mathrm{pc}^{-2}}{\Sigma_{\text {disc }}}\right)$

is $\tilde{Q}_{\text {thermal }}^{\text {un-shielded }} \gg 1$, i.e. the disc is fully stable. In this limit, we do not expect (nor see in our simulations) any significant star formation.

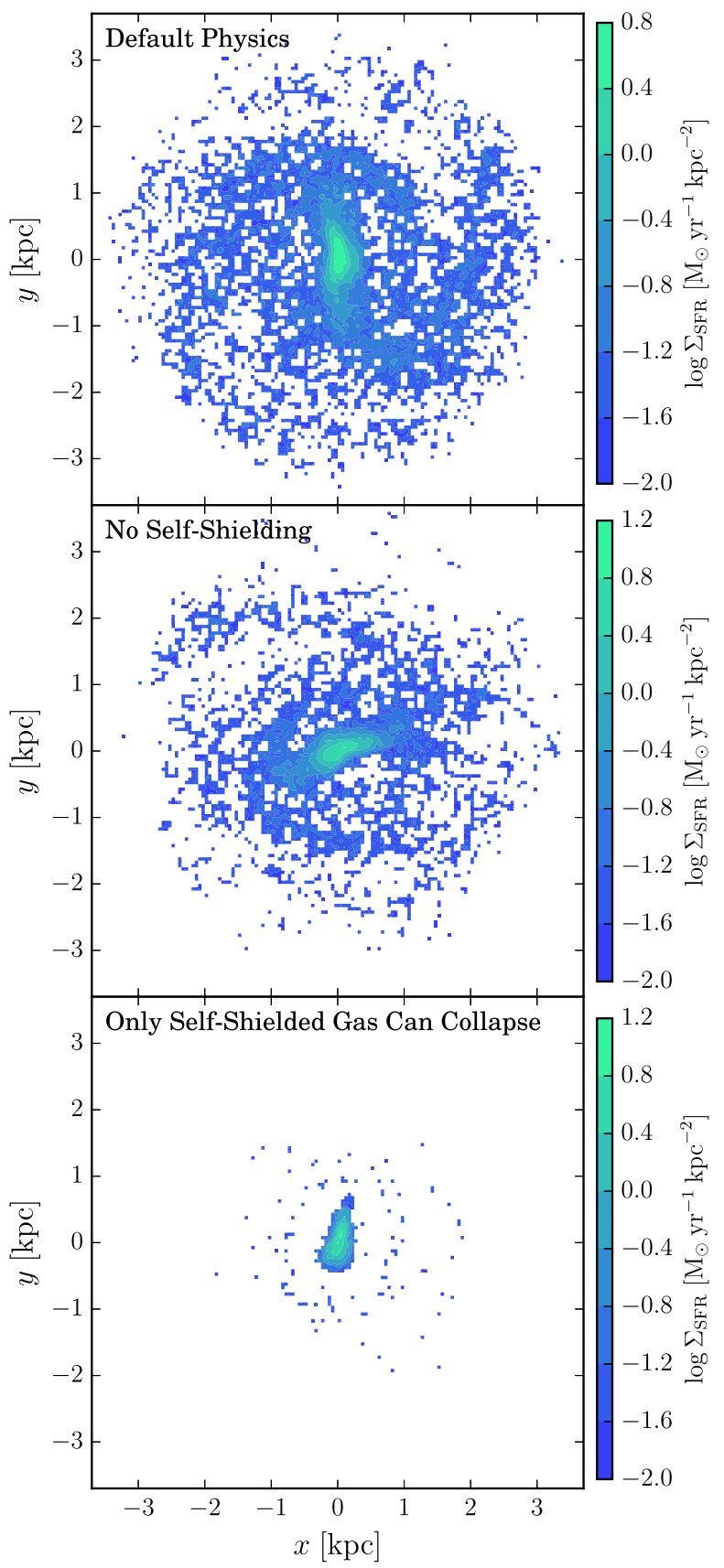

Figure 11. Demonstration of the importance of gravitational instability versus self-shielding in star formation. One Milky Way-mass simulation, m12i presented in Hopkins et al. (2014), was restarted from $z \approx 0.07$ and rerun with three sets of physics, the default physics implemented in the FIRE runs (top), one with both self-shielding and cooling below $10^{4} \mathrm{~K}$ disabled (middle), and one where shielded gas had normal properties but non-shielded gas had a large artificial pressure floor, effectively disabling gravitational fragmentation until the gas first became self-shielding (bottom). Coloured pixels indicate the extent and intensity of star formation since the runs were restarted in the form of the $500 \mathrm{Myr}$-averaged star formation rate surface density. The star formation largely has the same structure with or without self-shielding, as long as fragmentation is allowed (gas can still isothermally collapse and meet the star formation criteria). But when only annuli in a galaxy which are entirely self-shielding can fragment or collapse, only the central $\sim \mathrm{kpc}$ of the galaxy (where the gas is entirely molecular) efficiently forms stars. 
In the opposite limit (in e.g. the centres of massive galaxies), the surface densities are high, $\tilde{Q}_{\text {thermal }}^{\text {un-shield }} \ll 1$ (i.e. thermal support, even in the warm gas, is insufficient to stabilize the disc, so it is supersonically turbulent, with $Q \sim Q_{\text {turb }} \sim 1$ ), and the gas is selfshielding. In this limit, of course, we see efficient star formation.

What determines the transition between these two limits? It has been suggested that when an annulus reaches a critical column density to become self-shielding, it can suddenly cool to $T \ll 10^{4} \mathrm{~K}$, reducing the thermal pressure support of the gas disc against fragmentation (i.e. lowering the Toomre- $Q$ from $\gg 1$ to $\ll 1$ ) and so initiating gravitational collapse and star formation (e.g. Schaye 2004; Krumholz, McKee \& Tumlinson 2009a). This will occur when the dust optical depth $\tau=\Sigma_{\text {gas }} \kappa$ exceeds unity, or more accurately from Krumholz et al. (2009a), when $\Sigma_{\text {gas }} Z^{\prime}>27 \mathrm{M}_{\odot} \mathrm{pc}^{-2}$ (where $Z^{\prime} \equiv$ $\mathrm{Z} / \mathrm{Z}_{\odot}$ reflects the assumption of a constant dust-to-metals ratio).

Alternatively, an annulus which is not self-shielding (hence at a temperature $T \sim 10^{4} \mathrm{~K}$ ) will still become gravitationally unstable, when $\tilde{Q}_{\text {thermal }}^{\text {un-shielded }} \lesssim 1$, i.e. $\Sigma_{\text {disc }}\left(\Omega / \mathrm{Gyr}^{-1}\right)^{-1} \gtrsim 0.7 \mathrm{M}_{\odot} \mathrm{pc}^{-2}$. The annulus would then rapidly fragment isothermally (at $\sim 10^{4} \mathrm{~K}$ ) at first, until individual overdensities/fragments quickly become internally self-shielding (reaching local surface densities $\Sigma_{\text {gas }} Z^{\prime}>$ $27 \mathrm{M}_{\odot} \mathrm{pc}^{-2}$ as they collapse), then cool and fragment further to form stars. During this collapse, supersonic turbulence would be driven by gravitational instabilities and feedback to maintain a turbulent $Q \sim 1$, but the important point is that the thermal support $\left(\tilde{Q}_{\text {thermal }}^{\text {un-shielded }}\right)$ is insufficient.

The question is essentially which of these thresholds is reached 'first'. Fig. 8 examines this in our simulations by plotting all annuli in the space of $\tilde{Q}_{\text {thermal }}^{\text {un-shied }} \propto \Omega / \Sigma_{\text {disc }}$ versus $\tau_{\text {shielding }} \propto$ $\Sigma_{\text {gas }} Z$, and identifying those which are and are not star-forming. Clearly, robust star formation occurs in annuli which are not, on average, self-shielding (they have $\Sigma_{\text {gas }} Z^{\prime} \approx 1-5 \mathrm{M}_{\odot} \mathrm{pc}^{-2}$ ). We stress that the small sub-regions where star formation is occurring within those annuli are of course self-shielding (this is in fact required by our resolution-scale star formation model), and reach $\Sigma_{\text {gas }} Z^{\prime} \gg 100 \mathrm{M}_{\odot} \mathrm{pc}^{-2}$ locally. In contrast, the onset of star formation corresponds very closely to where $\tilde{Q}_{\text {thermal }}^{\text {un-shelded }} \approx 1$. This is consistent with observations of star-forming spiral galaxies by Martin \& Kennicutt (2001), who found that gravitational instability thresholds were sufficient to explain the extent of star-forming discs.

Examining Fig. 10 further, we see that the annuli all lie on a track which intercepts the instability threshold $\tilde{Q}_{\text {thermal }}^{\text {un-shed }} \approx 1$ more than a dex below the self-shielding threshold $\Sigma_{\text {gas }} Z \sim 27 \mathrm{M}_{\odot} \mathrm{pc}^{-2}$. Star formation (red pixels) is seen as annuli cross the instability line, and the distribution then turns upwards as star formation begins to enrich the annuli in $Z$ without much affecting $\Sigma_{\text {gas }}$ or $\tilde{Q}_{\text {thermal }}^{\text {un-shielded }}$ instantaneously. As annuli cross into the self-shielding regime, $\Sigma_{\text {gas }}$ quickly crosses into the high-surface-density regime, and vigorous star formation results in short depletion time-scales for these annuli, preventing highly shielded, low- $\tilde{Q}_{\text {thermal }}^{\text {un-shielded }}$ annuli from remaining long in that regime. Crossing into the self-shielded regime appears to coincide with the rapid rise in the lower envelope of $\dot{\Sigma}_{\star}-$ these regions are vigorously forming stars throughout and are unable to prevent themselves from cooling rapidly and fragmenting, as in Schaye (2004). Moreover, the high- $\tilde{Q}_{\text {thermal }}^{\text {un } \text { shield }} \gg 10$ annuli with very low $\Sigma_{\text {gas }} Z^{\prime}$ appear to come from the galactic outskirts at several times the half-mass radii.

To verify the relative importance of gravitational instability versus self-shielding, we also considered an idealized numerical experiment in Fig. 11. Specifically, we took one of our Milky Way-mass galaxy simulations (run m12i from Hopkins et al. 2014) and re-ran it for about $\sim 1$ Gyr close to $z=0$ (from $z=0.07$ to 0 ), modifying the physics in the re-run. We considered two cases.

(1) 'No self-shielding': In this case we disable self-shielding in our radiative heating routines and do not allow any cooling below $10^{4} \mathrm{~K} .{ }^{10}$ Clearly, Fig. 11 shows that gas is still able to fragment and form stars - the spatial extent of the star formation is nearly identical to our 'default' run, in fact, indicating that cooling to $T$ $\ll 10^{4} \mathrm{~K}$ is not what determines the outer cut-off of star formation in the disc (consistent with our argument in Fig. 10). The total star formation rate is also similar within 15 per cent.

(2) 'Only self-shielded gas can collapse': If self-shielding always preceded fragmentation and star formation, we should be able to disable Toomre-style fragmentation in gas which is not self-shielding, and obtain the same result. This is non-trivial in practice. We attempt to implement this as follows: for gas which is self-shielding (has cooled to $<8000 \mathrm{~K}$ and/or meets the Krumholz et al. (2009b) criterion), the physics is 'normal', but for gas which is not selfshielding, we add an artificial pressure term to the hydrodynamic equations $\left(P \rightarrow P_{\text {true }}+P_{\text {floor }}\right)$ where $P_{\text {floor }}=4 \times 10^{-11}\left(n / \mathrm{cm}^{-3}\right)$ (i.e. the pressure the gas would have at $3 \times 10^{5} \mathrm{~K}$ ). The specific value is chosen to ensure the non-shielded gas has an 'effective' Toomre$Q \sim$ a few (sufficient to prevent fragmentation but not 'blow up' the galaxy). When we do this, we see that efficient star formation becomes restricted to the central $\sim \mathrm{kpc}$ only (and the total star formation rate falls by a factor of $\approx 3$ ). This central region is basically the location of the molecular disc - i.e. the regime where the gas is entirely molecular, since that is where the disc is uniformly selfshielding. Clearly, this is not a good description of star formation in the 'default' simulation.

\subsubsection{Star formation in the SMC}

The star formation threshold behaviour seen in our simulations is consistent with observations of the SMC, as measured by Bolatto et al. (2011) and Hony et al. (2015). The star formation rates seen by Hony et al. (2015) agree well with the spatially resolved KS relation when considering young star counts as a measure of $\dot{\Sigma}_{\star}$. Moreover, considering the metallicity and surface density of the $\mathrm{SMC}$, in the SMC body/wing, $\tau \sim \Sigma_{\mathrm{gas}} \mathrm{Z} / \mathrm{Z}_{\odot} \sim 10 \mathrm{M}_{\odot} \mathrm{pc}^{-2}$, and in the SMC tail, $\Sigma_{\text {gas }} Z / \mathrm{Z}_{\odot} \sim 2 \mathrm{M}_{\odot} \mathrm{pc}^{-2}$ (Nidever, Majewski \& Butler Burton 2008; Oliveira 2009), the SMC body is thus not quite at the self-shielding threshold, and the tail is certainly not. On the other hand, when estimating $Q$ for the SMC wing, one finds $Q \sim 2 / 3$ and for the body $Q \ll 1$ [assuming a linearly rising rotation curve to $v_{\text {rot }} \sim 50 \mathrm{~km} \mathrm{~s}^{-1}$ at $R \sim 3.5 \mathrm{kpc}$ found by Stanimirović, StaveleySmith \& Jones (2004)]; hence, the SMC appears to be consistent with gravitational instabilities triggering star formation.

\section{CONCLUSIONS}

In this paper, we investigated the spatially resolved KS relation in the cosmological FIRE simulations with $z=0$ central halo masses ranging from $10^{10} \mathrm{M}_{\odot}$ to $10^{13} \mathrm{M}_{\odot}$. Our principal conclusions are the following:

\footnotetext{
${ }^{10}$ In our 'no self-shielding' run, we still enforce the Krumholz et al. (2009b) requirement described in Section 2 for whether an individual gas particle is allowed to form stars, since this is (strictly speaking) just a metallicitydependent local surface density threshold $\left(\Sigma_{\text {gas }} Z^{\prime}>27 \mathrm{M}_{\odot} \mathrm{pc}^{-2}\right)$ evaluated at the resolution scale.
} 
(i) The simulated galaxies exhibit a KS-like relation with slope and scatter consistent with observations. We emphasize that this relation emerges naturally rather than being imposed 'by hand' because we assume an instantaneous local star formation efficiency of 100 per cent per free-fall time, but the predicted global star formation efficiency is low, consistent with observations, due to stellar feedback. This is true across many orders of magnitude in halo mass and at all redshifts studied. The exact slope of the relationship between $\Sigma_{\text {SFR }}$ and $\Sigma_{\text {gas }}$ depends on the gas and star formation tracers used, but the two quantities are tightly correlated in all cases explored.

(ii) For the neutral hydrogen form of the relation, the simulations and observations agree well also in terms of normalization. In contrast, for the molecular hydrogen relation, the simulated and observed relations are systematically offset, likely because our proxy for the molecular gas (the 'cold and dense' tracer) underestimates the true molecular gas mass by $\sim 0.5$ dex for gas surface density $\lesssim$ $100 \mathrm{M}_{\odot} \mathrm{pc}^{-2}$.

(iii) The time-averaged KS relation does not appear to have a significant dependence on pixel size (i.e. map resolution) for gas surface densities with sufficiently resolved star formation rate distributions (i.e. above the $\Sigma_{\text {gas }}$ where the KS relation would yield at least a few young star particles per pixel given our mass resolution; see Section 2), with the slope of the power law remaining effectively unchanged. However, we are unable to resolve star formation rates at gas surface densities at our smallest pixel size (100 pc), for which observations exhibited large scatter in the KS relation $\left(\Sigma_{\text {gas }}\right.$ $\lesssim 10 \mathrm{M}_{\odot} \mathrm{pc}^{-2}$ ).

(iv) The KS relation and star formation efficiency in the FIRE simulations are independent of redshift. The simulations do not exhibit any metallicity-dependent cut-off; however, the star formation rate surface density is weakly dependent on the metallicity, on the order expected from SNe feedback's momentum injection dependence on metallicity (Cioffi et al. 1988; Martizzi et al. 2015).

(v) At the high end of gas surface density, where $\Sigma_{\text {gas }} \gtrsim 100 \mathrm{M}_{\odot}$ $\mathrm{pc}^{2}$ and gas is predominantly molecular, we find that the KS relation obeyed by the simulated galaxies is consistent with injection of momentum from supernovae balancing momentum dissipation in turbulence, or analogously, turbulent 'pressure' maintaining vertical hydrostatic equilibrium (Ostriker \& Shetty 2011; Faucher-Giguère et al. 2013; Hayward \& Hopkins 2015). This explanation yields a power law independent of redshift or metallicity at high gas surface densities, where $\dot{\Sigma}_{\star} \propto \Sigma_{\text {gas }} \Sigma_{\text {disc }}$ (see Section 4.1). Because the discs in our simulations are not particularly gas rich, we find a slightly steeper than linear KS relation in this regime.

(vi) In regions of low gas surface density $\left(\Sigma_{\text {gas }} \lesssim 10 \mathrm{M}_{\odot} \mathrm{pc}^{-2}\right)$, characteristically in galaxy outskirts and regions between spiral arms, our spatially resolved KS relation agrees well with that expected from a simple local equilibrium between photoheating from ionizing, or near-ionizing, radiation from young stars and radiative gas cooling. This argument yields a $\dot{\Sigma}_{\star} \propto Z \Sigma_{\text {gas }}^{2}$ power law (see Section 4.2), as discussed in Ostriker et al. (2010) and Hayward \& Hopkins (2015).

(vii) Vigorous star formation begins as gas self-gravity overcomes the gas thermal pressure gradient, thus making the gas Toomre-unstable. This self-gravity-driven collapse occurs around $\Sigma_{\text {gas }} \sim 1 \mathrm{M}_{\odot} \mathrm{pc}^{-2}$, an order of magnitude before the gas becomes self-shielding to UV radiation, at $\Sigma_{\text {gas }} \sim 27 \mathrm{M}_{\odot} \mathrm{pc}^{-2}$. Thus, we find that in the FIRE simulations, star formation is triggered by gravitational instabilities, which then cause the gas to fragment and collapse, thereby becoming self-shielding to ionizing radiation, cooling rapidly and forming stars. The threshold for gravitational instability, $Q \sim \Omega / \Sigma$, depends only on the density of gas and stars, i.e. the criterion for warm gas $\left(T \gtrsim 10^{4} \mathrm{~K}\right)$ to support itself thermally against fragmentation, and subsequently star formation, is independent of both $Z$ and $z$

Future observations with high spatial resolution and sensitivity to low surface brightnesses should aid in understanding the outskirts of galactic environments where star formation is on the brink of firing up and the surface densities of gas and stars are near the thresholds of gravitational instability and self-shielding. This will help determine if gravitational fragmentation - rather than selfshielding - is indeed the primary triggering mechanism of star formation. Similarly, future work implementing chemical networks and radiative transfer post-processing in cosmological simulations will help bridge the gap between simulated tracers of star formation and molecular gas and resolved observations.

\section{ACKNOWLEDGEMENTS}

MEO is grateful for the encouragement of his late father, SRO, in studying astrophysics, and for many helpful discussions with A. Wetzel, J. Schaye, S. Dib, and I. Escala. We are grateful to the anonymous referee for providing us with constructive comments and suggestions, which have significantly improved the work. This research has made use of NASA's Astrophysics Data System. MEO was supported by the National Science Foundation Graduate Research Fellowship under grant no. 1144469. CCH is grateful to the Gordon and Betty Moore Foundation for financial support. The Flatiron Institute is supported by the Simons Foundation. Support for PFH was provided by an Alfred P. Sloan Research Fellowship, NASA ATP Grant NNX14AH35G, and NSF Collaborative Research Grant \#1411920 and CAREER grant \#1455342. Numerical calculations were run on the Caltech compute cluster 'Zwicky' (NSF MRI award \#PHY-0960291) and allocations TG-AST120025, and TG-AST130039 granted by the Extreme Science and Engineering Discovery Environment (XSEDE) supported by the NSF. CAFG was supported by NSF through grants AST-1412836 and AST1517491, by NASA through grant NNX15AB22G, and by STScI through grants HST-AR-14293.001-A and HST-GO-14268.022-A. RF was supported by the Swiss National Science Foundation (grant no. 157591). DK acknowledges support from the NSF grant AST1412153 and Cottrell Scholar Award from the Research Corporation for Science Advancement. EQ was supported by NASA ATP grant 12-ATP12-0183, a Simons Investigator award from the Simons Foundation, and the David and Lucile Packard Foundation.

\section{REFERENCES}

Agertz O., Kravtsov A. V., 2015, ApJ , 824, 79

Agertz O., Kravtsov A. V., Leitner S. N., Gnedin N. Y., 2013, ApJ, 770, 25 Amorín R., Muñoz-Tuñón C., Aguerri J. A. L., Planesas P., 2016, A\&A, 588, A23

Asplund M., Grevesse N., Sauval A. J., Scott P., 2009, ARA\&A, 47, 481

Becerra F., Escala A., 2014, ApJ, 786, 56

Benincasa S. M., Wadsley J., Couchman H. M. P., Keller B. W., 2016, MNRAS, 462, 3053

Bigiel F., Leroy A., Walter F., Brinks E., de Blok W. J. G., Madore B., Thornley M. D., 2008, AJ, 136, 2846

Bigiel F., Leroy A., Walter F., Blitz L., Brinks E., de Blok W. J. G., Madore B., 2010, AJ, 140, 1194

Blanc G. A., Heiderman A., Gebhardt K., Evans N. J., II, Adams J., 2009, ApJ, 704, 842

Blitz L., Rosolowsky E., 2006, ApJ, 650, 933 
Bolatto A. D. et al., 2011, ApJ, 741, 12

Bolatto A. D., Wolfire M., Leroy A. K., 2013, ARA\&A, 51, 207

Bothwell M. S. et al., 2010, MNRAS, 405, 219

Bouché N. et al., 2007, ApJ, 671, 303

Calzetti D., Liu G., Koda J., 2012, ApJ, 752, 98

Chan T. K., Kereš D., Oñorbe J., Hopkins P. F., Muratov A. L., FaucherGiguère C.-A., Quataert E., 2015, MNRAS, 454, 2981

Cioffi D. F., McKee C. F., Bertschinger E., 1988, ApJ, 334, 252

Colín P., Avila-Reese V., Vázquez-Semadeni E., Valenzuela O., Ceverino D., 2010, ApJ, 713, 535

Daddi E. et al., 2010, ApJ, 714, L118

Dame T. M., Hartmann D., Thaddeus P., 2001, ApJ, 547, 792

Dib S., 2011, ApJ, 737, L20

Dib S., Hony S., Blanc G., 2017, MNRAS, 469, 1521

Dobbs C. L., 2015, MNRAS, 447, 3390

Downes D., Solomon P. M., 1998, ApJ, 507, 615

Elmegreen B. G., 1997, in Franco J., Terlevich R., Serrano A., eds, Rev. Mex. Astron. Astrofis. Ser. Conf. Vol. 6., 1st Guillermo Haro Conf. on Astrophys.: Starburst Activity in Galaxies., Puebla, Pue, Mexico. p. 165 Faucher-Giguère C.-A., Quataert E., Hopkins P. F., 2013, MNRAS, 433, 1970

Federrath C., Klessen R. S., 2012, ApJ, 761, 156

Feldmann R., Gnedin N. Y., 2011, ApJ, 727, L12

Feldmann R., Gnedin N. Y., Kravtsov A. V., 2011, ApJ, 732, 115

Feldmann R., Gnedin N. Y., Kravtsov A. V., 2012, ApJ, 758, 127

Feldmann R., Hopkins P. F., Quataert E., Faucher-Giguère C.-A., Kereš D., 2016, MNRAS, 458, L14

Forbes J. C., Krumholz M. R., Goldbaum N. J., Dekel A., 2016, Nature, 535,523

Freundlich J. et al., 2013, A\&A, 553, A130

Genzel R. et al., 2010, MNRAS, 407, 2091

Glover S. C. O., Clark P. C., 2012, MNRAS, 421, 9

Gnedin N. Y., Kravtsov A. V., 2011, ApJ, 728, 88

Gnedin N. Y., Tasker E. J., Fujimoto Y., 2014, ApJ, 787, L7

Grudić M. Y., Hopkins P. F., Faucher-Giguère C.-A., Quataert E., Murray N., Kereš D., 2018, MNRAS, 475, 3511

Hayward C. C. et al., 2014, MNRAS, 445, 1598

Hayward C. C., Hopkins P. F., 2015, MNRAS, 465, 1682

Hollenbach D. J., Tielens A. G. G. M., 1999, Rev. Mod. Phys., 71, 173

Hony S. et al., 2015, MNRAS, 448, 1847

Hopkins P. F. et al., 2017, preprint (arXiv:1702.06148)

Hopkins P. F., 2013, MNRAS, 428, 2840

Hopkins P. F., Quataert E., Murray N., 2011, MNRAS, 417, 950

Hopkins P. F., Quataert E., Murray N., 2012a, MNRAS, 421, 3488

Hopkins P. F., Quataert E., Murray N., 2012b, MNRAS, 421, 3522

Hopkins P. F., Cox T. J., Hernquist L., Narayanan D., Hayward C. C., Murray N., 2013a, MNRAS, 430, 1901

Hopkins P. F., Narayanan D., Murray N., 2013b, MNRAS, 432, 2647

Hopkins P. F., Kereš D., Murray N., Hernquist L., Narayanan D., Hayward C. C., 2013c, MNRAS, 433, 78

Hopkins P. F., Kereš D., Oñorbe J., Faucher-Giguère C.-A., Quataert E., Murray N., Bullock J. S., 2014, MNRAS, 445, 581

Hopkins P. F., Torrey P., Faucher-Giguère C.-A., Quataert E., Murray N., 2016, MNRAS, 458, 816

Hu C.-Y., Naab T., Glover S. C. O., Walch S., Clark P. C., 2017, MNRAS, 471,2151

Iffrig O., Hennebelle P., 2017, A\&A, 604, A70

Kennicutt R. C., Jr et al., 2007, ApJ, 671, 333

Kennicutt R. C., Jr, 1998, ApJ, 498, 541

Kennicutt R. C., Evans N. J., 2012, ARA\&A, 50, 531

Kim C.-G., Ostriker E. C., 2015, ApJ, 802, 99

Kim C.-G., Kim W.-T., Ostriker E. C., 2011, ApJ, 743, 25

Kim C.-G., Ostriker E. C., Kim W.-T., 2013, ApJ, 776, 1

Kraljic K., Renaud F., Bournaud F., Combes F., Elmegreen B., Emsellem E., Teyssier R., 2014, ApJ, 784, 112

Kroupa P., 2002, Science, 295, 82

Kruijssen J. M. D., Longmore S. N., 2014, MNRAS, 439, 3239

Krumholz M. R., Gnedin N. Y., 2011, ApJ, 729, 36
Krumholz M. R., Tan J. C., 2007, ApJ, 654, 304

Krumholz M. R., Thompson T. A., 2007, ApJ, 669, 289

Krumholz M. R., McKee C. F., Tumlinson J., 2009a, ApJ, 693 , 216

Krumholz M. R., McKee C. F., Tumlinson J., 2009b, ApJ, 699, 850

Kuhlen M., Krumholz M. R., Madau P., Smith B. D., Wise J., 2012, ApJ, 749,36

Lee E. J., Miville-Deschênes M.-A., Murray N. W., 2016, ApJ, 833, 229

Leitherer C. et al., 1999, ApJS, 123, 3

Leroy A. K. et al., 2013, AJ, 146, 19

Leroy A. K., Walter F., Brinks E., Bigiel F., de Blok W. J. G., Madore B., Thornley M. D., 2008, AJ, 136, 2782

Li Y., Mac Low M.-M., Klessen R. S., 2005, ApJ, 620, L19

Li Y., Mac Low M.-M., Klessen R. S., 2006, ApJ, 639, 879

Liu G., Koda J., Calzetti D., Fukuhara M., Momose R., 2011, ApJ, 735, 63

Ma X., Hopkins P. F., Wetzel A. R., Kirby E. N., Anglés-Alcázar D., FaucherGiguère C.-A., Kereš D., Quataert E., 2017, MNRAS, 467, 2430

Martin C. L., Kennicutt Jr. R. C., 2001, ApJ, 555, 301

Martizzi D., Faucher-Giguère C.-A., Quataert E., 2015, MNRAS, 450, 504

Martizzi D., Fielding D., Faucher-Giguère C.-A., Quataert E., 2016, MNRAS, 459, 2311

Mihos J. C., Hernquist L., 1994, ApJ, 437, 611

Murray N., 2011, ApJ, 729, 133

Murray N., Quataert E., Thompson T. A., 2010, ApJ, 709, 191

Narayanan D., Krumholz M. R., Ostriker E. C., Hernquist L., 2012, MNRAS, 421, 3127

Nidever D. L., Majewski S. R., Butler Burton W., 2008, ApJ, 679, 432

Oklopčić A., Hopkins P. F., Feldmann R., Kereš D., Faucher-Giguère C.-A., Murray N., 2017, MNRAS, 465, 952

Oliveira J. M., 2009, in Van Loon J. T., Oliveira J. M., eds, IAU Symp. 256, The Magellanic System: Stars, Gas, and Galaxies. Kluwer, Dordrecht, p. 191

Onodera S. et al., 2010, ApJ, 722, L127

Ostriker E. C., Shetty R., 2011, ApJ, 731, 41

Ostriker E. C., McKee C. F., Leroy A. K., 2010, ApJ, 721, 975

Padoan P., 1995, MNRAS, 277, 377

Papadopoulos P. P., Pelupessy F. I., 2010, ApJ, 717, 1037

Rafelski M., Gardner J. P., Fumagalli M., Neeleman M., Teplitz H. I., Grogin N., Koekemoer A. M., Scarlata C., 2016, ApJ, 825, 87

Richings A. J., Schaye J., 2016, MNRAS, 458, 270

Robertson B. E., Kravtsov A. V., 2008, ApJ, 680, 1083

Rownd B. K., Young J. S., 1999, AJ, 118, 670

Roychowdhury S., Huang M.-L., Kauffmann G., Wang J., Chengalur J. N., 2015, MNRAS, 449, 3700

Saitoh T. R., Daisaka H., Kokubo E., Makino J., Okamoto T., Tomisaka K., Wada K., Yoshida N., 2008, PASJ, 60, 667

Schaye J., 2004, ApJ, 609, 667

Schaye J., Dalla Vecchia C., 2008, MNRAS, 383, 1210

Schmidt M., 1959, ApJ, 129, 243

Schruba A., Leroy A. K., Walter F., Sandstrom K., Rosolowsky E., 2010, ApJ, 722, 1699

Scoville N. et al., 2016, ApJ, 820, 83

Semenov V. A., Kravtsov A. V., Gnedin N. Y., 2016, ApJ, 826, 200

Shapiro K. L. et al., 2010, MNRAS, 402, 2140

Shetty R., Ostriker E. C., 2012, ApJ, 754, 2

Shetty R., Kelly B. C., Bigiel F., 2013, MNRAS, 430, 288

Shetty R., Kelly B. C., Rahman N., Bigiel F., Bolatto A. D., Clark P. C., Klessen R. S., Konstandin L. K., 2014a, MNRAS, 437, L61

Shetty R., Clark P. C., Klessen R. S., 2014b, MNRAS, 442, 2208

Silk J., 1997, ApJ, 481, 703

Solomon P. M., Vanden Bout P. A., 2005, ARA\&A, 43, 677

Solomon P. M., Downes D., Radford S. J. E., Barrett J. W., 1997, ApJ, 478 144

Sparre M., Hayward C. C., Feldmann R., Faucher-Giguère C.-A., Muratov A. L., Kereš D., Hopkins P. F., 2015, MNRAS, 466, 88

Springel V., Hernquist L., 2003, MNRAS, 339, 289

Stanimirović S., Staveley-Smith L., Jones P. A., 2004, ApJ, 604, 176

Strong A. W., Mattox J. R., 1996, A\&A, 308, L21 
Su K.-Y., Hopkins P. F., Hayward C. C., Faucher-Giguere C.-A., Keres D., Ma X., Robles V. H., 2016, MNRAS, 471, 144

Tacconi L. J. et al., 2013, ApJ, 768, 74

Thompson T. A., Quataert E., Murray N., 2005, ApJ, 630, 167

Toomre A., 1964, ApJ, 139, 1217

Torrey P., Hopkins P. F., Faucher-Giguère C.-A., Vogelsberger M., Quataert E., Kereš D., Murray N., 2016, MNRAS, 467, 2301

Verley S., Corbelli E., Giovanardi C., Hunt L. K., 2010, A\&A, 510, A64

Wada K., Norman C. A., 2007, ApJ, 660, 276

Wei L. H., Vogel S. N., Kannappan S. J., Baker A. J., Stark D. V., Laine S., 2010, ApJ, 725, L62

Williams J. P., McKee C. F., 1997, ApJ, 476, 166

Wong T., Blitz L., 2002, ApJ, 569, 157

\section{APPENDIX A: ROBUSTNESS OF STAR FORMATION RATES TO VARIATIONS IN STAR FORMATION, COOLING, AND STELLAR FEEDBACK}

Here, we demonstrate the robustness of the star formation rate in the FIRE simulations to reasonable changes in the implemented star formation, cooling, and stellar feedback physics (reviewed in detail in Hopkins et al. 2014). A number of previous studies have consistently demonstrated the convergence of star formation rates and the KS relation, with resolution and numerical implementations of star formation (Saitoh et al. 2008; Federrath \& Klessen 2012; Hopkins et al. 2012b, 2013a,b,c, 2016; Agertz et al. 2013). In Fig. A1, we illustrate this with a set of simple tests using the newest version of the code (part of a more general numerical study, presented in detail in Hopkins et al. 2017). In each case, we re-start the same Milky Way-mass simulation (m12i from Hopkins et al. 2014, as in Section 4.3 in the text), and re-run it from $z=0.07-0$ with different numerical choices. This ensures the initial conditions are identical; $\Sigma_{\text {gas }}$ for the galaxy, for example, is fixed, so we can simply read off from the star formation rate whether the galaxy's location in the KS law would change.

We compare our default star formation model, using the criteria enumerated in Section 2, here with $n_{\text {crit }}=1000 \mathrm{~cm}^{-3}$ and $\epsilon_{\mathrm{sf}}=1$, where $\epsilon_{\mathrm{sf}}$ represents the local efficiency with which gas turns into stars in a free-fall time, i.e. $\dot{\rho}_{\star}=\epsilon_{\mathrm{sf}} \rho_{\mathrm{mol}} / t_{\mathrm{ff}}$, to variations with $\epsilon_{\mathrm{sf}}$ $=0.01-100, n_{\text {crit }}=5-1000 \mathrm{~cm}^{-3}$, and turning on/off the additional virial and molecular criteria. We find that the star formation rate (and indeed the entire spatially resolved $\mathrm{KS}$ relation) is effectively the same in all cases.

The gas in the restarts responds dynamically to these changes in the star formation prescription, as seen in Fig. A2. Variations in $n_{\text {crit }}$ allow gas to evolve to higher/lower densities before turning into stars rapidly, seen in the rapid fall-off of the densest gas in the various models. Changes in $\epsilon_{\mathrm{sf}}$ yield similar results, with smaller $\epsilon_{\text {sf }}$ values allowing gas to continue evolving to higher physical densities. Removing the virial and molecular criteria appears to have the same effect as lowering the density threshold or raising the local star formation efficiency, likely as more of the gas just above the threshold is converted to stars rapidly that is not necessarily bound. All the while, the star formation rates in the restarts are essentially unchanged; we see that the gas in the galaxies is dynamically evolving to produce the 'correct' star formation rate to regulate itself. Detailed observations of the gas density CDF in the Milky Way and nearby galaxies may thus help constrain sub-grid star formation prescriptions to produce realistic gas density distributions, without altering the overall star formation rates in the simulations.

In Fig. A1, we also vary our cooling model, replacing all lowtemperature cooling physics with a single cooling rate, putting all gas on a single, solar-metallicity cooling curve, and removing the molecular star formation requirement. We see that there is no effect on the star formation rate; we similarly find no effect on the outflow rate or global morphology. Details of the phase structure, of course, differ, but these have no large dynamical effect, consistent with various previous studies that have found that almost all gas in galaxies is supersonically turbulent and has cooling times much shorter than their dynamical times (Hopkins, Quataert \& Murray 2011, 2012a; Glover \& Clark 2012).

We also explicitly consider the mass resolution convergence by up- and down-sampling the particle distribution with particle splitting/merging. We find that the star formation rate is nearly identical over $\sim 2.5$ dex in mass resolution, even a factor of $\sim 10$ lower resolution compared to our 'standard FIRE' resolution. This is consistent with our argument in Section 2 that we only need to marginally resolve the Toomre scale to achieve convergence in the star formation rate because the most massive clouds dominate star formation (Williams \& McKee 1997).

As seen in the bottom left-hand panel of Fig. A1, variation in the strength of feedback per mass of young stars is the only effective means of changing the star formation rate. As galaxies self-regulate for a given level of feedback, changing the strength of feedback systematically results in higher star formation rates for lower levels of feedback per star and vice versa. This is consistent with our scalings in Section 4.

\section{APPENDIX B: VARIOUS MOLECULAR GAS MASS PROXIES IN THE FIRE SIMULATIONS}

As compared to the neutral gas (atomic and molecular) mass, predicting the molecular mass in the FIRE simulations alone is difficult, as cooling and self-shielding are calculated using approximate look-up tables and are not done fully self-consistently with radiative transfer at the particle scale (see Hopkins et al. 2014, for details of the numerical implementation of cooling and shielding effects in FIRE-1). For reasons presented in Appendix A, getting the cooling and shielding even grossly incorrect in the coolest, densest gas generally has no dynamical effect on the simulations but does greatly affect the high-density tail of the gas volumetric-density distribution. Similarly, the gas density threshold for star formation and the instantaneous local star formation efficiency assumed affect the time that gas spends at the highest resolvable densities (see Fig. A2). What truly constitutes molecular gas in the simulations, or what would be observed as such, requires careful forward modelling of the chemical abundances and molecular line emission. That, coupled with large uncertainties in the gas phase structure at low temperatures and its dependence on the aforementioned grid/particle-scale star formation and cooling prescriptions, leaves us with crude, though physically motivated, proxies for molecular gas masses in the simulations. In this work, we have used a conservative estimator for molecular gas, our cold and dense $\left(T<300 \mathrm{~K}\right.$ and $\left.n_{\mathrm{H}}>10 \mathrm{~cm}^{-3}\right)$ gas tracer. We compare this explicitly with two other empirical estimators for molecular gas at the kpc-scale, and one other local (e.g. few pc) estimate, in Fig. B1. There, we compare an estimator from Leroy et al. (2008), for which the molecular fraction is taken to be directly proportional to the mid-plane pressure of the ISM, which is calculated using both the gas and stellar surface densities and dynamical times (see Blitz \& Rosolowsky 2006, from which they adapt their empirical estimator), and fits for the molecular fraction from Krumholz et al. (2009b) relating to local dust opacity applied both at the pixel $(\mathrm{kpc})$ and at the particle $(\mathrm{pc})$ scale. 


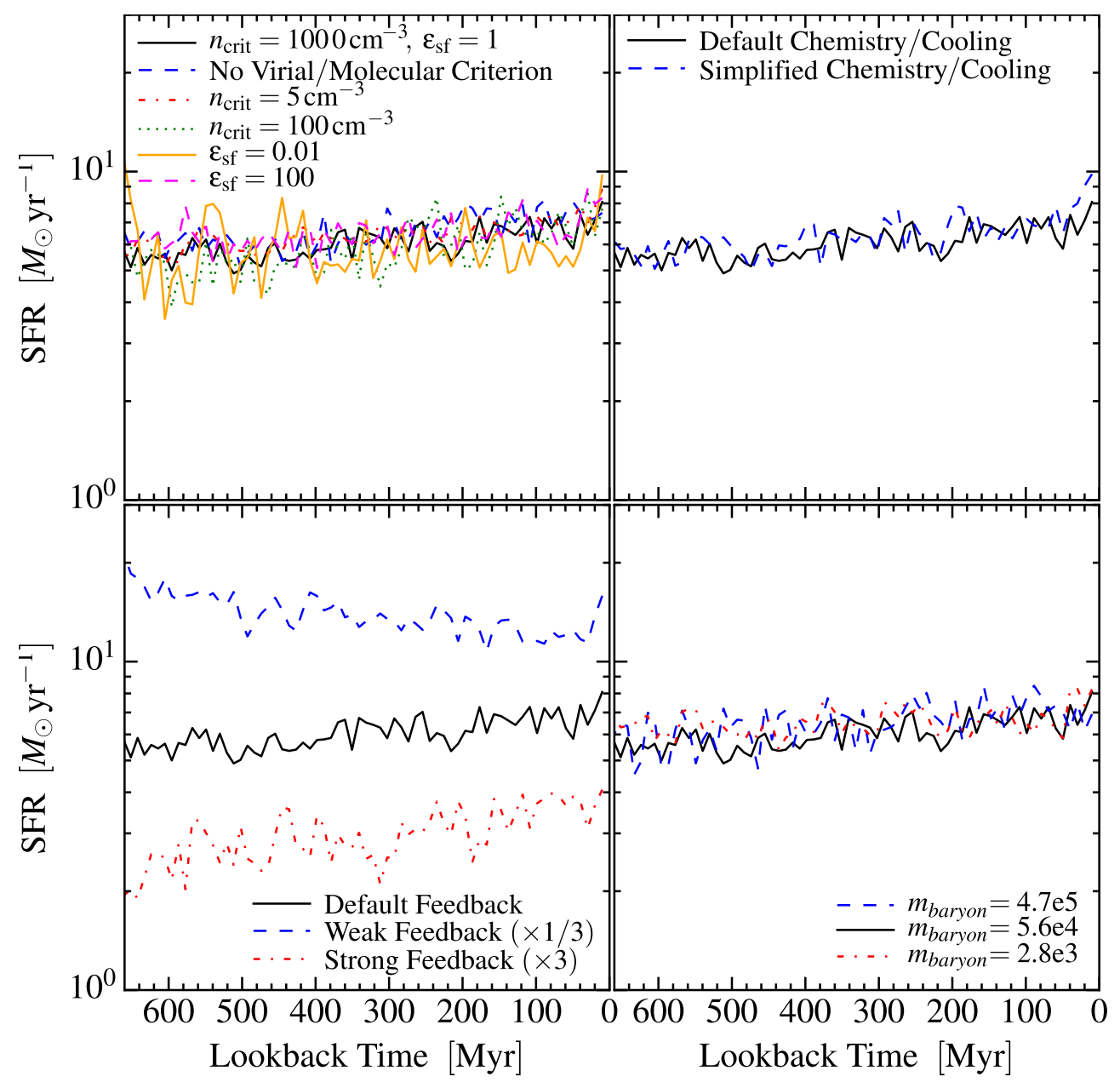

Figure A1. Star formation rate versus time in our MW-mass $(\mathbf{m} 12 \mathbf{i})$ simulation from redshift $z \approx 0.07$ to 0 ; the simulation was restarted at $z=0.07$ and run with varying parameters to study the effect on the star formation rate given the same initial galaxy properties. Top left: Effect of the resolution-scale star formation criteria. In our 'default' model, gas that is self-gravitating, molecular, and dense $\left(n>n_{\text {crit }}=1000 \mathrm{~cm}^{-3}\right)$ forms stars at a rate $\dot{\rho}=\epsilon_{\mathrm{sf}} \rho_{\mathrm{mol}} / t_{\mathrm{ff}}$, with $\epsilon_{\mathrm{sf}}=1$. We compare (1) removing the self-gravity and molecular restrictions, (2-3) varying $n_{\text {crit }}$, and (4-5) varying $\epsilon_{\text {sf. }}$ Bottom left: We vary the strength of feedback by multiplying/dividing the rates of all mechanisms per unit stellar mass by 3 relative to the predictions from the stellar evolution models. Top right: Default physical cooling model compared with a toy model that ignores all low-temperature cooling physics and puts all gas on a single, solar-metallicity cooling curve. Bottom right: Resolution effects, changing the baryonic particle mass. The results of this figure show that only the strength of feedback significantly alters the star formation rate at fixed $\Sigma_{\text {gas }}$; varying the sub-grid star formation law has essentially no effect.

The two empirical estimators lie roughly 0.5 dex above the cold and dense gas tracer at all gas surface densities and are in better concordance with observations, uncertainties in them notwithstanding (see Appendix C). Due to the steepness of the Krumholz et al. (2009b) fitting function at the atomic-to-molecular transition, $\sim 10 \mathrm{M}_{\odot} \mathrm{pc}^{-2}$, very few kpc-scale pixels contribute to the data shown (because many kpc-scale pixels have $\log \Sigma_{\mathrm{H}_{2}} \ll-1$ ), indicating the necessity of assuming clumping factors when applying these fits on scales larger than GMCs themselves in low-gas surface density environments (e.g. disc outskirts). This $\sim 0.5$ dex discrepancy indicates three things: (1) the FIRE simulations do appear to produce correct SFRs for the large-scale pressure of the ISM, (2) the SFRs are in concordance with those expected given the largescale optical depths of the ISM, and (3) the FIRE-1 simulations appear to either produce insufficient high-density gas or consume high-density gas more quickly than expected. Points (1) and (2) lend credence to trusting the large-scale structure and dynamics of the ISM and the FIRE simulations; however, point (3) indicates that we have not yet converged on producing a realistic phase struc- ture of the ISM at the highest densities near our resolution limits (noting that in FIRE-1 the gas density threshold for star formation is $\sim 50 \mathrm{~cm}^{-2}$, quite low compared to the densities of PDRs and the critical density of ${ }^{12} \mathrm{CO}$ Hollenbach \& Tielens 1999). We compared several other estimators for the molecular fraction, including a less-stringent temperature cut $(T<3000 \mathrm{~K})$ and a stellar surface density fit $\left(\Sigma_{\mathrm{H}_{2}} / \Sigma_{\mathrm{HI}} \propto \Sigma_{\star}\right)$ also explored in Leroy et al. (2008), but omit them for clarity as they all lay between the extremal values of the cold and dense tracer at the low end and the Leroy et al. (2008)/Blitz \& Rosolowsky (2006) empirical pressure and Krumholz et al. (2009b) opacity fits at the high end. Reconstructing the $f_{\mathrm{H}_{2}}$ fraction of the particles themselves using the fits of Krumholz \& Gnedin (2011) used in GIZMO (also seen in the figure), however, agrees more closely (underestimating only by $\sim 0.1$ dex) with the cold and dense gas tracer. The fact that the same estimator applied at the pc- and kpc-scales can produce results with 0.5-0.7 dex differences likely owes to point (3) and the difficulties in estimating the local column depths for shielding. Throughout the main body of this paper, we use the cold and dense tracer as a 


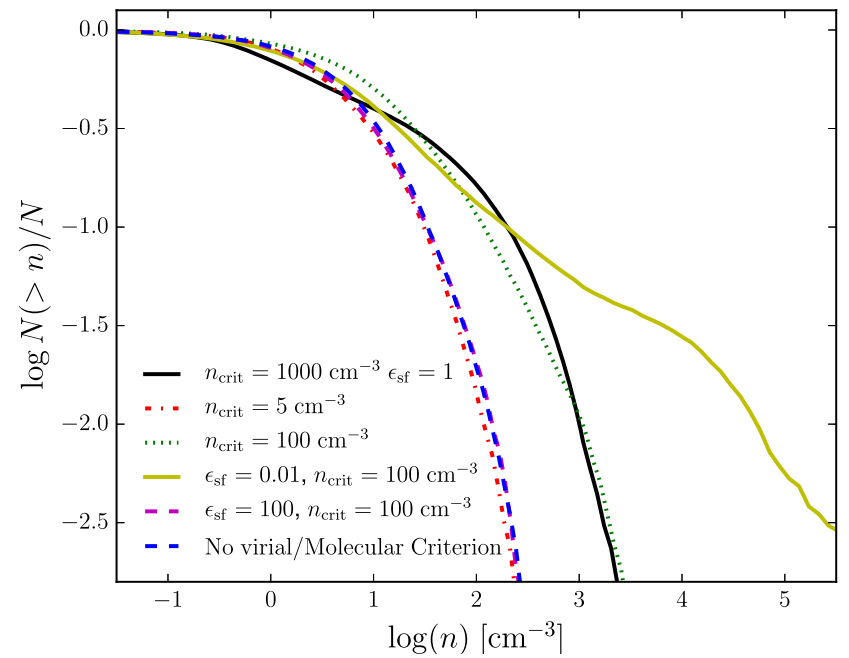

Figure A2. Gas number density CDF in our MW-mass (m12i) simulation at redshift $z \approx 0$ for the various star formation model test runs in the upper left-hand panel of Fig. A1. The CDFs evolve dynamically such that the 'correct' amount of dense gas forms to support the required SFR to regulate the galaxy. In our (new) 'default' model, with the highest $n_{\text {crit }}=1000 \mathrm{~cm}^{-3}$ more gas evolves to higher densities before turning into stars, compared to the other SF models except for our low-efficiency run. Removing the virial and molecular thresholds is nearly equivalent to drastically reducing $n_{\text {crit }}$ (it had $n_{\text {crit }}=100 \mathrm{~cm}^{-3}$ ) or increasing $\epsilon$. Intuitively, holding $\dot{\rho}_{\star}$ constant in equation (2), i.e. the SFRs converge to the 'necessary' value, we expect that $n \propto \epsilon^{-1 / 2}$. Indeed, we see that increasing $\epsilon$ by a factor of 100 moves the gas density CDF a dex towards lower densities.

lower limit on the molecular gas column and acknowledge an $\sim 0.5$ dex uncertainty in our dense gas tracer, dependent on our choice of proxy in order to most fairly show the range of tension between our results and observations given that choice. In Figs 2, 3, and 5, we use arrows to indicate how shifting the molecular gas surface densities based on the cold and dense tracer 0.5 dex higher would bring the simulations and observations in closer agreement.

We explicitly compare the approximate molecular fraction versus neutral gas surface density relation obtained using the cold and dense gas proxy $\left(T<300 \mathrm{~K}\right.$ and $\left.n_{\mathrm{H}}>10 \mathrm{~cm}^{-3}\right)$ to fits from Krumholz et al. (2009b), with 1 kpc pixels. A plot of $\Sigma_{C \& D}$, the cold and dense gas surface density, versus $\Sigma_{\text {neut }}$, the total neutral gas $\left(\mathrm{HI}+\mathrm{H}_{2}\right)$ surface density for gas with approximately solar metallicity $\left(Z_{\odot} \pm 0.1\right.$ dex $)$, is shown in Fig. B2. Compared to the steep atomic-to-molecular transition thresholds found by Krumholz et al. (2009b), the ratio $\Sigma_{\mathrm{C} \& \mathrm{D}} / \Sigma_{\text {neut }}$ converges much more slowly to unity (only near $\sim 100 \mathrm{M}_{\odot} \mathrm{pc}^{-2}$ ). The bulk of pixels ( $\sim 70$ per cent) lie below $f_{\mathrm{H}_{2}}=0.33$, thus indicating that the cold and dense gas tracer is likely consistently underestimating the molecular fraction by $0.5-1$ dex for $\Sigma_{\text {gas }}>10 \mathrm{M}_{\odot} \mathrm{pc}^{-2}$. For gas surface densities between 1 and $10 \mathrm{M}_{\odot} \mathrm{pc}^{-2}$, it is unclear how much of the high values for the molecular fractions at low gas surface densities is due to beam-filling (i.e. cloud-counting) effects, and it is unclear whether the cold and dense gas tracer is over or underestimating the molecular fractions there.

\section{APPENDIX C: UNCERTAINTY IN $X_{\text {CO }}$ FOR OBSERVED $\Sigma_{\mathrm{H}_{2}}$ AND TENSION WITH SIMULATIONS}

All of the observations to which we compare our results infer molecular hydrogen masses from $\mathrm{CO}$ emission using a single or bimodel

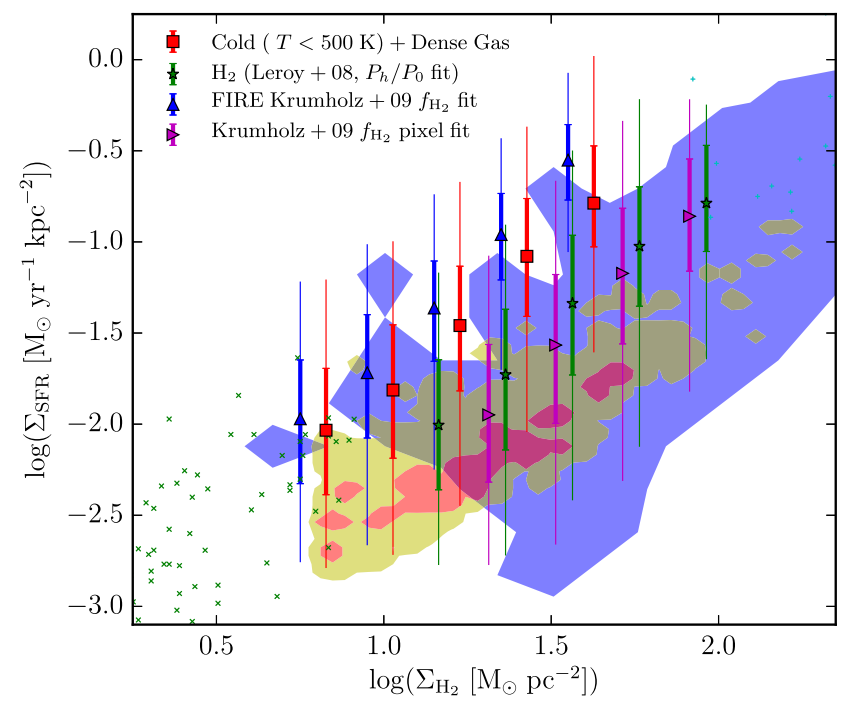

Figure B1. Comparison of three of proxies for the molecular gas surface density in the $\mathrm{KS}$ relation at $1 \mathrm{kpc}^{2}$ from a subset of the galaxy simulations presented in this work from $z \approx 0.2$ to 0 . Points, error bars, and shaded regions (molecular KS observations) are in the style of Fig. 2. The cold and dense $\left(<300 \mathrm{~K}\right.$ and $\left.>10 \mathrm{~cm}^{-3}\right)$ tracer is calculated on a per-particle basis, whereas the $\mathrm{H}_{2}$ masses predicted by an empirical fit from Leroy et al. (2008) and the molecular fraction fits of Krumholz et al. (2009b) are produced from kpc-averaged quantities of the mapped pixels, and applied directly to the gas particles themselves as is done in calculating the SFRs in FIRE. The relations obtained when the Leroy et al. (2008) and Krumholz et al. (2009b) fitting functions are applied to predict the molecular gas surface density at the pixel scale are more consistent with observations than when the cold and dense tracer is used, or when the Krumholz et al. (2009b) fit is applied at the particle scale, because at a fixed SFR surface density, these tracers yield molecular gas surface densities $\sim 0.5$ dex lower than those obtained using the two aforementioned fitting functions. This result suggests that the FIRE simulations are producing 'correct' SFRs given the large-scale properties of the ISM (mid-plane pressure and dust opacity), but insufficient gas is able to remain in or reach the highest resolvable densities in the simulations. Other proxies for the molecular gas mass, including a warmer temperature cut $(3000 \mathrm{~K})$, yield molecular fractions between the extremal cases presented here. These results suggest an $\sim 0.5$ dex uncertainty in our estimates of molecular gas surface density, with the cold and dense tracer systematically biased low.

CO-to- $\mathrm{H}_{2}$ conversion factor, $X_{\mathrm{CO}}$, which is used to convert from $\mathrm{CO}$ linewidth $\mathrm{W}\left({ }^{12} \mathrm{C}^{16} \mathrm{O} J=1 \rightarrow 0\right)$ to $\mathrm{H}_{2}$ column density $\mathrm{N}\left(\mathrm{H}_{2}\right)$ using the following relation:

$\mathrm{N}\left(\mathrm{H}_{2}\right)=X_{\mathrm{CO}} \mathrm{W}\left({ }^{12} \mathrm{C}^{16} \mathrm{O} J=1 \rightarrow 0\right)$.

The value of $X_{\mathrm{CO}}$ is of the order of $10^{20} \mathrm{~cm}^{-2} /\left(\mathrm{K} \mathrm{km} \mathrm{s}^{-1}\right.$ ) (see Bolatto et al. 2013, for a review on the $X_{\mathrm{CO}}$ conversion factor), but there is tremendous disagreement about the exact value it takes and its dependences on surface density, metallicity, and other parameters. As a result, we find it necessary to understand the extent to which the observational data can vary for differing, but reasonable, assumptions about $X_{\mathrm{CO}}$. Fig. $\mathrm{C} 1$ shows how various choices for the value of $X_{\mathrm{CO}}$ affect the tension between observations and our 'standard' tracers of atomic + molecular $\left(\Sigma_{\mathrm{HI}+\mathrm{H}_{2}}\right)$ and cold and dense $\left(\sim \Sigma_{\mathrm{H}_{2}}\right)$ gas surface density. We compare three conversion factors: (left column) a 'star-forming disc' $X_{\mathrm{CO}}=2 \times 10^{20} \mathrm{~cm}^{-2} /\left(\mathrm{K} \mathrm{km} \mathrm{s}^{-1}\right)$, a value widely adopted for low-redshift observations of Milky Waylike galaxies (Strong \& Mattox 1996; Dame, Hartmann \& Thaddeus 2001; Bigiel et al. 2008; Genzel et al. 2010; Shapiro et al. 2010; Wei et al. 2010; Tacconi et al. 2013; Amorín et al. 2016); (middle 


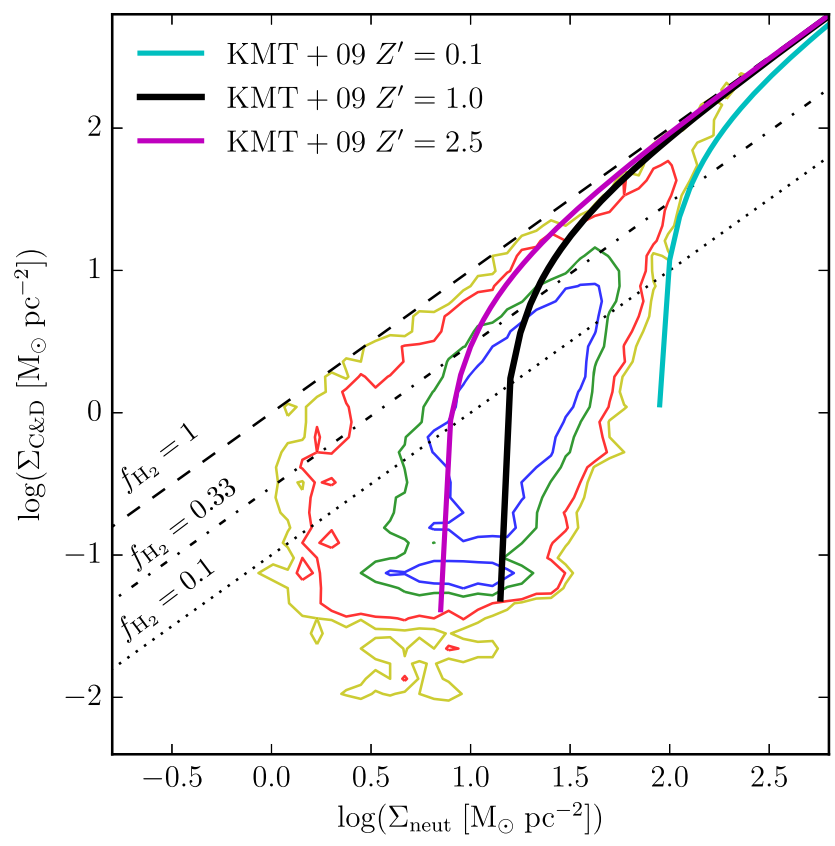

Figure B2. Distribution of $\Sigma_{\mathrm{C} \& \mathrm{D}}$, the cold and dense gas surface density, versus $\Sigma_{\text {neut }}$, the neutral gas surface density, for gas with metallicities $-0.1<\log Z / Z_{\odot}<0.1\left(Z \approx Z_{\odot}\right)$ in the FIRE simulations, with pixel sizes of $1 \mathrm{kpc}$. Coloured (yellow, red, green, blue) contours indicate $(95,90$, 70,50 )th-percentile-inclusion contours of the data. Black (dashed, dashdotted, dotted) lines represent $f_{\mathrm{H}_{2}}=(1,0.33,0.1)$. Coloured lines (cyan, black, magenta) represent $f_{\mathrm{H}_{2}}$ molecular fraction fits for various metallicities $\left(Z / Z_{\odot}=0.1,1.0,2.5\right)$ from Krumholz et al. $(2009 b)$. The core of the 'molecular fraction' (as represented by $\Sigma_{\mathrm{C} \& \mathrm{D}} / \Sigma_{\text {neut }}$ ) has a steeply rising slope between $0.5<\log \Sigma_{\text {neut }}<1.0$. However, the cold and dense fraction does not converge to unity as quickly as the fits from Krumholz et al. (2009b) at solar metallicities and has a tail of high fractions to lower gas surface densities, below their metallicity-dependent thresholds.

column) a 'starburst' $X_{\mathrm{CO}}=X_{\mathrm{CO} \text {, disc }} / 3.2$ which is a factor of 3.2 smaller than the disc conversion factor, owing to the fact that at high gas surface densities in extreme star-forming systems the disc $X_{\mathrm{CO}}$ predicts gas masses in excess of observed dynamical masses, which is a known problem for ULIRG observations (Solomon et al. 1997; Downes \& Solomon 1998; Solomon \& Vanden Bout 2005; Bothwell et al. 2010); and (right column) a variable $X_{\mathrm{CO}}$ interpolation function based on Narayanan et al. (2012). We take the form of the Narayanan et al. (2012) interpolation function to be

$X_{\mathrm{CO}}=\min \left[4,6.75 \times W_{\mathrm{CO}}^{-0.32}\right] \times 10^{20} \frac{\mathrm{cm}^{-2}}{\mathrm{~K} \mathrm{~km} \mathrm{~s}^{-1}}$,

which is identical to that presented in their work, ${ }^{11}$ assuming a Solar gas metallicity (see Ostriker \& Shetty 2011, for a compara- ble interpolation function). We recalibrate all of the observations enumerated in Section 2.1 for the KS relation using the $X_{\mathrm{CO}}$ value predicted using equation (C2). To correct the $\Sigma_{\mathrm{HI}+\mathrm{H}_{2}}$ measurements, we decomposed the total column into atomic and molecular components (the latter then being corrected in the manner of the $\Sigma_{\mathrm{H}_{2}}$ values) using data from the references themselves, where available, or assuming a molecular fraction fit from Leroy et al. (2008) when necessary.

In Appendix B, we demonstrated that the ratio of cold and dense tracer to the neutral hydrogen surface density slowly converges to one above $\sim 10 \mathrm{M}_{\odot} \mathrm{pc}^{-2}$ and hovers $\sim 0.5$ dex below other empirical fits and the neutral gas surface density in the KS plane until $\gtrsim 100 \mathrm{M}_{\odot} \mathrm{pc}^{-2}$. Considering this, adopting the disc $X_{\mathrm{CO}}$ leads to large disagreement at the highest gas surface densities, which we believe our molecular gas surface density proxy is nearly converged for (and other studies of the FIRE simulations have shown that the centres of our Milky Way-mass galaxies are not outliers in terms of gas surface density or star formation rate; Hopkins et al. 2014, 2016; Torrey et al. 2016), whereas a purely 'starburst' $X_{\mathrm{CO}}$ seems to suggest our simulations are overpredicting neutral gas surface densities by $\sim 0.5$ dex everywhere but at the most extreme gas surface densities. Given that there is little support for either of these values of $X_{\mathrm{CO}}$ holding for all gas surface densities, it is reasonable to use an interpolation function, such as that of Narayanan et al. (2012), for the range of observations. Otherwise, between the disc and starburst $X_{\mathrm{CO}}$ factors an $\sim 0.5$ dex uncertainty exists, before even considering reasonable additional factor of a few differences in those values themselves (Bolatto et al. 2013). This level of variation is of the order of the difference between extremal estimators of our molecular gas masses (see the difference between the cold and dense tracer and the Krumholz et al. 2009b relation applied to individual pixels shown in Fig. B1). Although the cold and dense gas tracer is clearly a conservative estimate of the molecular gas mass in the simulations and more careful forward-modelling of $\mathrm{CO}$ emission is clearly necessary (motivating a future work), the uncertainty in the observational value of $X_{\mathrm{CO}}$ makes it difficult to determine the absolute level of (dis)agreement between observations and simulations (all simulations, not just the FIRE simulations) at the $\sim 0.5-1 \mathrm{dex}$ level. As a result, in order to attempt to compare the results from FIRE on an appropriate footing with the observations throughout this paper, which both cover a parameter space of star formation rates and gas surface densities in both the 'star-forming disc' and 'starburst' regimes, we recalibrate the compiled observations with the Narayanan et al. (2012) $X_{\mathrm{CO}}$ interpolation function.

\footnotetext{
${ }^{11}$ It is noted that their normalization/maximum $X_{\mathrm{CO}}$ is twice that of the 'star-forming disc' $X_{\mathrm{CO}}$ factor.
} 


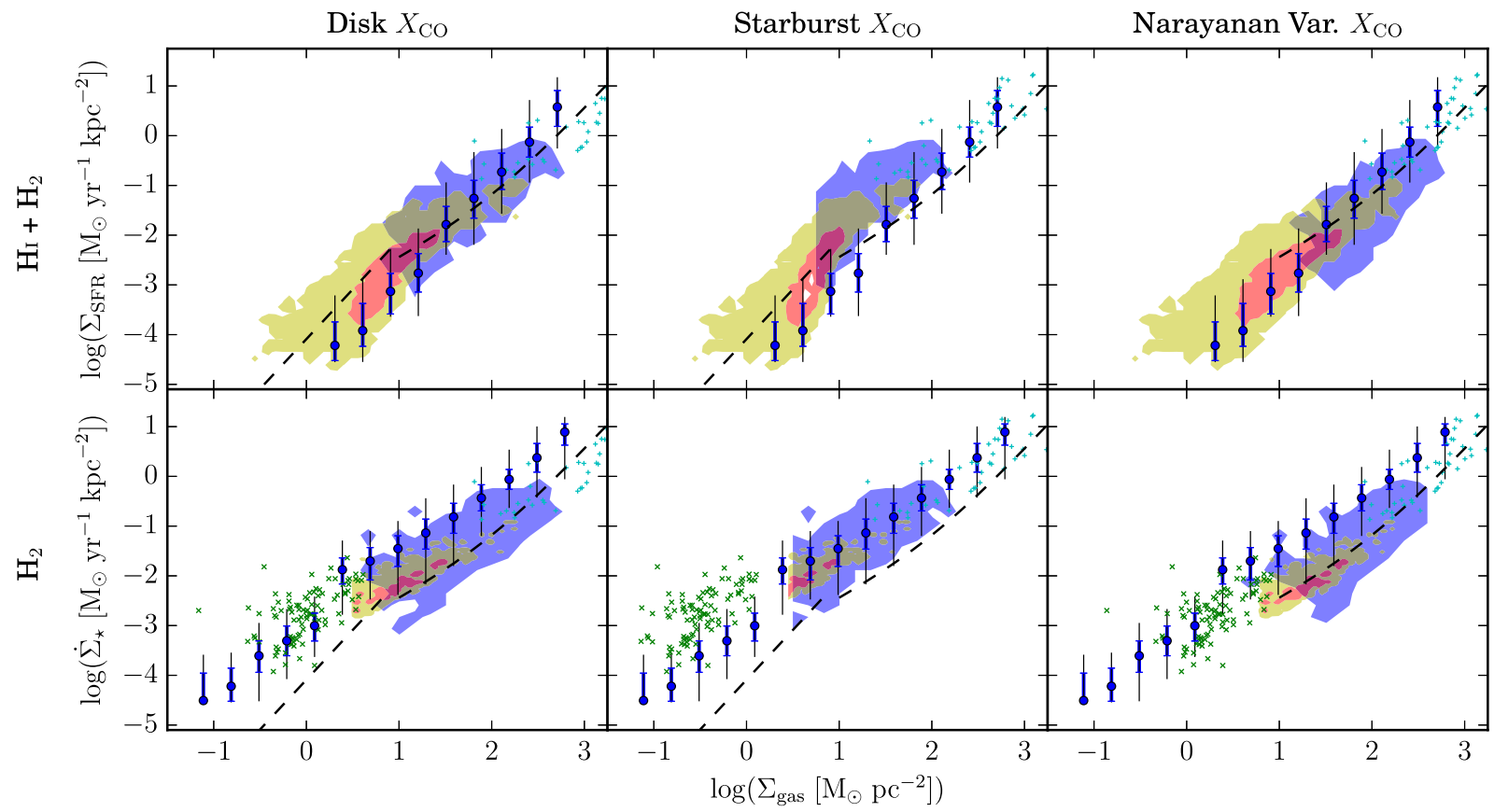

Figure C1. KS relation in the FIRE runs in $1 \mathrm{kpc}^{2}$ pixels, binned by $\Sigma_{\text {gas }}$, for neutral and 'molecular' gas tracers (rows) and the $10 \mathrm{Myr}$-averaged star formation rate surface density, with three different $X_{\mathrm{CO}}$ conversion factors applied to observations (enumerated in Fig. 2) for comparison (columns). Atomic + molecular hydrogen is $\sim \Sigma_{\mathrm{HI}+\mathrm{H}_{2}}$ (top row), and cold and dense gas includes particles with $T<300 \mathrm{~K}$ and $n_{\mathrm{H}}>10 \mathrm{~cm}^{-3}\left(\sim \Sigma_{\mathrm{H}_{2}}\right.$, bottom row). All observations have been re-calibrated with either a standard 'star-forming disc' $X_{\mathrm{CO}}=2 \times 10^{20} \mathrm{~cm}^{-2} /\left(\mathrm{K} \mathrm{km} \mathrm{s}^{-1}\right)$ (left column, as adopted by Bigiel et al. 2008), a 'starburst' $X_{\mathrm{CO}}=X_{\mathrm{CO} \text {, disc }} / 3.2$ (middle column, adopted by Genzel et al. 2010), and a variable $X_{\mathrm{CO}}$ fit interpolating between the 'star-forming disc' and 'starburst' $X_{\mathrm{CO}}$ values (right column, normalized by a factor of 2 higher than either of the other $X_{\mathrm{CO}}$ 's) found by Narayanan et al. (2012), as described in Section 2.1. The neutral gas observations have been decomposed into constituent $\Sigma$ and $\Sigma_{\mathrm{H}_{2}}$ columns, with the latter being corrected (see Section 2.1). Uncertainty in values of $X_{\mathrm{CO}}$, allowing for $\sim 0.5$ dex variations in observationally inferred molecular gas masses, can affect the (dis)agreement between FIRE and observations, on the same order as variations in choices of molecular gas proxy in our mapping (see Fig. B1). We adopt the Narayanan et al. (2012) variable $X_{\mathrm{CO}}$ interpolation function throughout the main text.

This paper has been typeset from a $\mathrm{T}_{\mathrm{E}} \mathrm{X} / \mathrm{L} \mathrm{A} \mathrm{E} \mathrm{X}$ file prepared by the author. 